\title{
TRANSLATES OF EXPONENTIAL BOX SPLINES AND THEIR RELATED SPACES
}

\author{
ASHER BEN-ARTZI AND AMOS RON
}

\begin{abstract}
Exponential box splines ( $E B$-splines) are multivariate compactly supported functions on a regular mesh which are piecewise in a space $\mathscr{H}$ spanned by exponential polynomials. This space can be defined as the intersection of the kernels of certain partial differential operators with constant coefficients.

The main part of this paper is devoted to algebraic analysis of the space $\mathbf{H}$ of all entire functions spanned by the integer translates of an $E B$-spline. This investigation relies on a detailed description of $\mathscr{H}$ and its discrete analog $\mathscr{S}$. The approach taken here is based on the observation that the structure of $\mathscr{H}$ is relatively simple when $\mathscr{H}$ is spanned by pure exponentials while all other cases can be analyzed with the aid of a suitable limiting process. Also, we find it more efficient to apply directly the relevant differential and difference operators rather than the alternative techniques of Fourier analysis. Thus, while generalizing the known theory of polynomial box splines, the results here offer a simpler approach and a new insight towards this important special case.

We also identify and study in detail several types of singularities which occur only for complex $E B$-splines. The first is when the Fourier transform of the $E B$-spline vanishes at some critical points, the second is when $\mathscr{H}$ cannot be embedded in $\mathscr{S}$ and the third is when $\mathbf{H}$ is a proper subspace of $\mathscr{H}$. We show, among others, that each of these three cases is strictly included in its former and they all can be avoided by a refinement of the mesh.
\end{abstract}

1. Introduction and the main results. The first part of this paper is primarily concerned with the analysis of an $s$-dimensional space $\mathscr{H}$ which is intimately related to the so-called "exponential box splines". In general $\mathscr{H}$ is the intersection of the kernels of a certain set of partial differential operators with constant coefficients. In the univariate case this set contains one ordinary differential operator. We prove that $\mathscr{H}$ is always finite dimensional and is spanned by products of polynomials by exponentials so that in this sense the multivariate setting generalizes the univariate case. In addition to the continuous case described above we investigate a sequence space $\mathscr{S}$ which is the discrete analogue of $\mathscr{H}$. This space is defined by the parallel partial difference operators.

In the second part of the paper we give a detailed algebraic analysis of exponential box splines, based on the results on $\mathscr{H}$ and $\mathscr{S}$, focusing on the space of entire functions spanned by the integer translates of an exponential box spline and the pre-image of this space. The analysis is carried out with an intensive aid of the

Received by the editors August 6, 1987.

1980 Mathematics Subject Classification (1985 Revision). Primary 41A63, 41A15; Secondary $33 \mathrm{~A} 10$. 
differential and difference operators mentioned above and essentially avoids Fourier analysis which is usually applied to similar problems.

In the sequel we first introduce the exponential box splines and review some of their elementary properties. Then the spaces $\mathscr{H}$ and $\mathscr{S}$ are defined and some of the main results are presented. Finally we give the outline of the paper and conclude the introduction with several notations and conventions.

In the following ||$,\langle\rangle$, , denote cardinality, linear span and scalar product respectively. The exponential function $\exp (\underline{\theta} \cdot \underline{x})$ is abbreviated as $e_{\underline{\theta}}(\underline{x})$.

Let $\Gamma$ be a finite set consisting of pairs (not necessarily distinct) of the form

$$
\gamma=\left(\underline{x}_{\gamma}, \lambda_{\gamma}\right), \quad \underline{x}_{\gamma} \in \mathbb{Z}^{s} \backslash\{\underline{0}\}, \lambda_{\gamma} \in \mathbb{C} .
$$

Let $h$ be a positive scaling parameter. The exponential box spline based on $\Gamma$ is a distribution which is defined via its Fourier transform as

$$
\hat{B}_{h}(\Gamma \mid \underline{x})=\prod_{\gamma \in \Gamma} \int_{0}^{h} e^{\left(\lambda_{\gamma}-i \underline{x}_{\gamma} \cdot \underline{x}^{\prime} t\right.} d t .
$$

It is known, $\left[\mathbf{R}_{1}\right]$, that under the assumption

$$
\left\langle\left\{\underline{x}_{\gamma}\right\}_{\gamma \in \Gamma}\right\rangle=\mathbb{R}^{s}
$$

$B_{h}(\Gamma \mid \cdot)$ is a compactly supported function. Its support is included in (and usually equals) the convex polyhedron

$$
R_{h}(\Gamma):=\left\{\sum_{\gamma \in \Gamma} t_{\gamma} \underline{x}_{\gamma} \mid 0 \leq t_{\gamma} \leq h \forall \gamma \in \Gamma\right\} .
$$

When $\lambda_{\gamma}=0$, all $\gamma, B_{h}(\Gamma \mid \cdot)$ is a piecewise polynomial function and is termed "a polynomial box spline" or simply "a box spline". Polynomial box splines were introduced by de Boor and DeVore, [BD], and investigated by de Boor and Höllig $[\mathbf{B H}]$, Dahmen and Micchelli $\left[\mathbf{D M}_{1-3}\right]$, Jia $\left[\mathbf{J}_{1,2}\right]$ and others. The general exponential box splines were introduced in $\left[\mathbf{R}_{1,2}\right]$.

Let us now present the distribution space and the sequence space which are studied herein. Denote for $\underline{x} \in \mathbb{R}^{s}$ by $D_{\underline{x}}$ the distributional directional derivative in the $\underline{x}$-direction and by the $E_{\underline{x}}$ the shift operation defined by

$$
E_{\underline{x}} f \rightarrow f(\cdot-\underline{x}) \text {. }
$$

Every $K \subset \Gamma$ is associated with the following partial differential and difference operators

$$
\begin{gathered}
D^{K}:=\prod_{\gamma \in K}\left(D_{\underline{x}_{\gamma}}-\lambda_{\gamma}\right), \\
\nabla_{h}^{K}:=\prod_{\gamma \in K}\left(I-e^{h \lambda_{\gamma}} E_{h \underline{x}_{\gamma}}\right),
\end{gathered}
$$

where $I$ is the identity mapping. Here $D^{K}$ acts on $\mathscr{D}^{\prime}\left(\mathbb{R}^{s}\right)$, the space of all complex valued $s$-dimensional distributions and $\nabla_{h}^{K}$ acts on both $\mathscr{D}^{\prime}\left(\mathbb{R}^{s}\right)$ and space of all sequences defined on the lattice

$$
\mathbb{Z}_{h}^{s}:=\left\{\underline{\alpha} \in \mathbb{R}^{s} \mid h^{-1} \underline{\alpha} \in \mathbb{Z}^{s}\right\} .
$$


Now the distribution space corresponding to $\Gamma$ is defined as

$$
\mathscr{H}(\Gamma)=\left\{f \in \mathscr{D}^{\prime}\left(\mathbb{R}^{s}\right) \mid D^{K} f \equiv 0, \forall K \subset \Gamma, \ni\left\langle\left\{\underline{x}_{\gamma}\right\}_{\gamma \in \Gamma \backslash K}\right\rangle \neq \mathbb{R}^{s}\right\},
$$

while the discrete space is defined analogously as

$$
\mathscr{S}_{h}(\Gamma)=\left\{u: \mathbb{Z}_{h}^{s} \mid \nabla_{h}^{K} u \equiv 0, \forall K \subset \Gamma, \ni\left\langle\left\{\underline{x}_{\gamma}\right\}_{\gamma \in \Gamma \backslash K}\right\rangle \neq \mathbb{R}^{s}\right\} .
$$

It can be shown, $\left[\mathbf{R}_{1}\right]$, that for every $K \subset \Gamma$ and $f \in \mathscr{D}^{\prime}\left(\mathbb{R}^{s}\right)$

$$
\nabla_{h}^{K} f=B_{h}(K) * D^{K} f .
$$

The last formula allows us to produce sequences in $\mathscr{S}_{h}(\Gamma)$ by restricting elements of $\mathscr{H}(\Gamma) \cap C\left(\mathbb{R}^{s}\right)$ to the lattice points. This close relation between the two spaces is used here to bound the dimension of $\mathscr{H}(\Gamma)$ in terms of the dimension of $\mathscr{S}_{h}(\Gamma)$.

The following sets, independently introduced in $\left[\mathbf{D M}_{2}\right]$ and $\left[\mathbf{J}_{2}\right]$, are useful for local analysis of $\mathscr{S}_{h}(\Gamma)$ :

$$
b_{h}^{\Gamma}(\underline{x})=\left\{\underline{\alpha} \in \mathbb{Z}_{h}^{s} \mid \underline{x}-\underline{\alpha} \in R_{h}(\Gamma)\right\} .
$$

$b_{h}^{\Gamma}(\cdot)$ can be viewed as a map from $\mathbb{R}^{s}$ to subsets of $\mathbb{Z}_{h}^{s}$. Every maximal open set where $b_{h}^{\Gamma}(\cdot)$ is constant is termed $a \Gamma-h$ cell. The union of all cells is denoted by $A_{h}(\Gamma)$. It is easy to see that $A_{h}(\Gamma)$ is open and dense in $\mathbb{R}^{s}$. Also, it is known $\left[\mathbf{R}_{1}\right]$, that the exponential box spline $B_{h}(\Gamma)$ and each of its translates by $\underline{\alpha} \in \mathbb{Z}_{h}^{s}$ are infinitely differentiable on $A_{h}(\Gamma)$.

Another set which plays an important role in the investigation of $\mathscr{H}(\Gamma)$ and $\mathscr{S}_{h}(\Gamma)$ is the set of all "bases" in $\Gamma$

$$
\mathbf{J}(\Gamma)=\left\{J \subset \Gamma|| J \mid=s,\left\langle\left\{\underline{x}_{\gamma}\right\}_{\gamma \in J}\right\rangle=\mathbb{R}^{s}\right\} .
$$

Given $J \in \mathbf{J}(\Gamma)$ the notation $X_{J}$ stands for the $s \times s$ matrix whose columns are $\left\{\underline{x}_{\gamma}\right\}_{\gamma \in J}$.

Since spaces of functions on $\mathbb{R}^{s}$ which are spanned by exponential polynomials frequently occur in this paper, we find it convenient to refer to such spaces as " $P E$ spaces". Analogously the restriction of a " $P E$ space" to the lattice $\mathbb{Z}_{h}^{s}$ is called "a $P E$ sequence space".

The main results on $\mathscr{H}(\Gamma)$ and $\mathscr{S}_{h}(\Gamma)$ are summarized in the following theorem.

THEOREM 1.1. Assume $\left\langle\left\{\underline{x}_{\gamma}\right\}_{\gamma \in \Gamma}\right\rangle=\mathbb{R}^{s}$. Then

(a) $\mathscr{H}(\Gamma)$ and $\mathscr{S}_{h}(\Gamma)$ are finite dimensional $P E$ spaces.

(b) $\operatorname{dim} \mathscr{H}(\Gamma)=|\mathbf{J}(\Gamma)|$.

(c) $\operatorname{dim} \mathscr{S}_{h}(\Gamma)=\sum_{J \in \mathbf{J}(\Gamma)}\left|\operatorname{det} X_{J}\right|$.

(d) For any $\underline{x} \in A_{h}(\Gamma)$ the set $b_{h}^{\Gamma}(\underline{x})$ is unisolvent for interpolation by elements of $\mathscr{S}_{h}(\Gamma)$ namely any data on $b_{h}^{\Gamma}(\underline{x})$ is uniquely interpolated by $\mathscr{S}_{h}(\Gamma)$.

We also derive a necessary and sufficient condition for the existence of a unique interpolant $f \in \mathscr{H}(\Gamma)$ to every $u(\cdot) \in \mathscr{S}_{h}(\Gamma)$.

In the case $\lambda_{\gamma}=0$ all $\gamma$ (= the polynomial case), the results stated in Theorem 1.1 are due to Dahmen and Micchelli $\left[\mathbf{D M}_{1-3}\right]$. In particular, the polynomial case of Theorem 1.1(b) was proved in $\left[\mathbf{D M}_{2}\right]$ by simultaneous induction on $|\Gamma|$ and $s$. The method taken here is essentially different: we establish first Theorem 1.1 for the "splitting" case (termed simple) in which $\mathscr{H}(\Gamma)$ is spanned by pure exponentials. The passage to the general case is executed by a unified limiting process primarily based on the following theorem. 
THEOREM 1.2. Let $L$ be a positive number and $n$ a positive integer. Denote by $E(n, L)$ the set of all s-dimensional functions of the form $\sum_{j=1}^{n} c_{j} e_{\underline{\theta}^{j}}(\underline{x})$, where $c_{1}, \ldots, c_{n}$ are complex numbers and the exponents $\underline{\theta}^{1}, \ldots, \underline{\theta}^{n}$ satisfy the following condition: $\max _{1 \leq r \leq s ; 1 \leq j \leq n}\left|\underline{\theta}_{r}^{j}\right|<L$. Let $\left\{H_{j}\right\}_{j=1}^{\infty} \subset E(n, L)$ be a sequence of linear spaces. Then there exists a subsequence $\left\{H_{j_{l}}\right\}_{l=1}^{\infty}$ and a function space $H$ such that

(a) $\operatorname{dim} H=\min \operatorname{dim} H_{j}=: d$.

(b) Every $f \in H$ is a limit in the $C^{\infty}\left(\mathbb{R}^{s}\right)$ topology of a sequence $\left\{f_{j_{l}} \mid f_{j_{l}} \in H_{j_{l}} \forall l\right\}$.

The fact that $\mathscr{H}(\Gamma)$ is closed under differentiation $\left(D\right.$-invariant) and $\mathscr{S}_{h}(\Gamma)$ is closed under translates by $\underline{\alpha} \in \mathbb{Z}_{h}^{s}$ (shift-invariant) is of fundamental significance here. The following theorem gives a characterization of a finite dimensional space which satisfies such invariance properties.

THEOREM 1.3. (a) Every finite dimensional D-invariant distribution space is a PE space.

(b) Every finite dimensional shift-invariant sequence space is a PE sequence space.

Theorem 1.3(a) can also be stated with $D$-invariance replaced by translationinvariance. Thus, for the space of tempered distributions rather than $\mathscr{D}^{\prime}\left(\mathbb{R}^{s}\right)$ this result follows directly from the Whitney-Schwartz theorem, $\left[\mathbf{S}_{2}\right.$, Theorem VI], which says that every closed translation-invariant space of tempered distributions contains a dense $P E$ subspace. Yet, the analogous results to the Whitney-Schwartz theorem for other spaces are usually not valid. E.g., for $C^{\infty}\left(\mathbb{R}^{s}\right)$ the claim is true only for $s=1$ (see $\left.\left[\mathbf{S}_{1}, \mathbf{G}\right]\right)$.

In $\S 2$ we prove Theorem 1.2 and apply this theorem to show that

$$
\operatorname{dim} \mathscr{H}(\Gamma) \geq|\mathbf{J}(\Gamma)| .
$$

$\S 3$ is devoted to the proof of Theorem 1.3. In $\S 4$ it is first shown that $\mathscr{S}_{h}(\Gamma)$ and $\mathscr{H}(\Gamma)$ are finite dimensional $P E$ spaces. Then their structure is analyzed in detail with special emphasis to the "simple" case. The proof of Theorem 1.1 is completed in $\S 5$.

Substituting $f=B_{h}(\Gamma \backslash K)$ in (1.11) one obtains the identity, [ $\left.\mathbf{R}_{1}\right]$,

$$
\nabla_{h}^{K} B_{h}(\Gamma \backslash K)=D^{K} B_{h}(\Gamma), \quad \forall K \subset \Gamma .
$$

This simple formula is our basic tool in the investigation of exponential box splines made in $\S 6$. There we define for $u: \mathbb{Z}_{h}^{s}$

$$
B_{h}(\Gamma) *_{h} u:=\sum_{\underline{\alpha} \in \mathbb{Z}_{h}^{s}} u(\underline{\alpha}) B_{h}(\Gamma \mid \cdot-\underline{\alpha}),
$$

and view $B_{h}(\Gamma) *_{h}$ as an operation from the space of all sequences defined on $\mathbb{Z}_{h}^{s}$. Our aim is to characterize the space $\mathbf{H}_{h}(\Gamma)$ of all entire functions in the range of $B_{h}(\Gamma) *_{h}$. The main relations between $\mathbf{H}_{h}(\Gamma), \mathscr{H}(\Gamma), \mathscr{S}_{h}(\Gamma)$ and $B_{h}(\Gamma) *_{h}$ are as follows.

THEOREM 1.4 .
(a) $\mathbf{H}_{h}(\Gamma) \subset \mathscr{H}(\Gamma)$
(b) $B_{h}(\Gamma) *_{h}$ maps $\mathscr{S}_{h}(\Gamma)$ onto $\mathbf{H}_{h}(\Gamma)$. 
The analysis of $\mathbf{H}_{h}(\Gamma)$ is completed in $\S 7$ where we establish necessary and sufficient conditions for equality in (1.17) and derive a formula for $\operatorname{dim} \mathbf{H}_{h}(\Gamma)$. To describe some of the results of that section denote:

$$
\Theta(\Gamma)=\left\{\underline{\theta} \in \mathbb{C}^{s} \mid \exists J \in \mathbf{J}(\Gamma), \ni \underline{x}_{\gamma} \cdot \underline{\theta}-\lambda_{\gamma}=0 \forall \gamma \in J\right\} .
$$

Then we have

THEOREM 1.5. Consider the following three conditions:

(a) $\hat{B}_{h}(\Gamma \mid-i \underline{\theta}) \neq 0, \forall \underline{\theta} \in \Theta(\Gamma)$.

(b) $h\left(\underline{\theta}^{1}-\underline{\theta}^{2}\right) \notin 2 \pi i \mathbb{Z}^{s} \backslash \underline{0}, \forall \underline{\theta}^{1}, \underline{\theta}^{2} \in \Theta(\Gamma)$.

(c) $\mathbf{H}_{h}(\Gamma)=\mathscr{H}(\Gamma)$.

Then $(\mathrm{a}) \Rightarrow(\mathrm{b}) \Rightarrow(\mathrm{c})$.

It is also proved that in the univariate case conditions (b), (c) of Theorem 1.5 are equivalent. Yet, with the aid of a bivariate counterexample we show that in the multivariate case the implication (c) $\Rightarrow$ (b) is not valid.

In this paper we consistently use standard multivariate notations. Thus for $\underline{\alpha} \in \mathbb{Z}^{s}, \underline{x} \in \mathbb{R}^{s}$ we use $|\underline{\alpha}|=\alpha_{1}+\cdots+\alpha_{s}, D^{(\underline{\alpha})}=\partial^{|\underline{\alpha}|} / \partial x_{1}^{\alpha_{1}} \cdots \partial x_{s}^{\alpha_{s}}$ and $\underline{x} \underline{\alpha}=\prod_{j=1}^{s} x_{j}^{\alpha_{j}}$. The space of all $s$-dimensional polynomials is denoted as usual by $\pi$. Also we find it convenient to denote for every $K \subset \Gamma$

$$
X_{K}:=\left\{\underline{x}_{\gamma}\right\}_{\gamma \in K}, \quad \underline{\lambda}_{K}:=\left\{\lambda_{\gamma}\right\}_{\gamma \in K}, \quad\langle K\rangle:=\left\langle X_{K}\right\rangle .
$$

A vector $\underline{x}_{\gamma}$ (with $\gamma \in \Gamma$ ) is sometimes treated as the pair $\left(\underline{x}_{\gamma}, 0\right)$. So $D^{X_{K}}=$ $\prod_{\gamma \in K} D_{\underline{x}_{\gamma}}, B_{h}\left(X_{K}\right)$ is the (polynomial) box spline based on $\left(X_{K}, \underline{0}\right)$ etc. When concerning a set of a single element we usually omit brackets; thus $D^{\gamma}, \nabla_{h}^{\underline{x}}, \Gamma \backslash \gamma$ are used rather than the rigorous notations $D^{\{\gamma\}}, \nabla_{h}^{\left\{\underline{x}_{\gamma}\right\}}, \Gamma \backslash\{\gamma\}$. Whenever it is possible we may identify a function space with its restriction to the lattice points. Finally, to simplify the definition of $\mathscr{H}(\Gamma)$ and $\mathscr{S}_{h}(\Gamma)$ we denote

$$
\mathscr{K}(\Gamma):=\left\{K \subset \Gamma \mid\langle\Gamma \backslash K\rangle \neq \mathbb{R}^{s}\right\} .
$$

With the aid of this notation we may write

$$
\mathscr{H}(\Gamma)=\bigcap_{K \in \mathscr{H}(\Gamma)} \operatorname{ker} D^{K}, \quad \mathscr{S}_{h}(\Gamma)=\bigcap_{K \in \mathscr{K}(\Gamma)} \operatorname{ker} \nabla_{h}^{K} .
$$

2. A lower bound for $\operatorname{dim} \mathscr{H}(\Gamma)$. We make here the first step in the analysis of $\mathscr{H}(\Gamma)$ and $\mathscr{S}_{h}(\Gamma)$ by showing that

$$
\operatorname{dim} \mathscr{H}(\Gamma) \geq|\mathbf{J}(\Gamma)| .
$$

In case $\underline{\lambda}_{\Gamma}=\underline{0}$, this result is Theorem 2.2 in $\left[\mathbf{D M}_{2}\right]$ which was proved there by induction on $|\Gamma|$ and $s$. The method used here differs from that of $\left[\mathbf{D M}_{2}\right]$. First we prove (2.1) for "simple" defining sets and then apply Theorem 1.2 to extend the results to general $\Gamma$ 's. The proof of Theorem 1.2 is postponed to the end of this section.

Given a defining set $\Gamma$ and $\underline{\theta} \in \mathbb{C}^{s}$ denote

$$
\Gamma_{\underline{\theta}}=\left\{\gamma \in \Gamma \mid \underline{x}_{\gamma} \cdot \underline{\theta}-\lambda_{\gamma}=0\right\},
$$

and define (compare with (1.18))

$$
\Theta(\Gamma)=\left\{\underline{\theta} \in \mathbb{C}^{s} \mid\left\langle\Gamma_{\underline{\theta}}\right\rangle=\mathbb{R}^{s}\right\} .
$$


The elements of $\Theta(\Gamma)$ will be referred to as "nodes". Note that for $\gamma \in \Gamma$ and $\underline{\theta} \in \mathbb{C}^{s}, D^{\gamma} e_{\underline{\theta}}(\cdot) \equiv 0$ if and only if $\gamma \in \Gamma_{\underline{\theta}}$. Now, for $\underline{\theta} \in \Theta(\Gamma)$ and $K \in \mathscr{K}(\Gamma)$ one has by definition $\langle\Gamma \backslash K\rangle \neq \mathbb{R}^{s}$ and consequently (2.3) implies $K \cap \Gamma_{\underline{\theta}} \neq \varnothing$. Thus

$$
D^{K} e_{\underline{\theta}}=D^{K \backslash \Gamma_{\underline{\theta}}}\left(D^{K \cap \Gamma_{\underline{\theta}}} e_{\underline{\theta}}\right) \equiv 0,
$$

and we conclude

Proposition 2.1. For each $\underline{\theta} \in \Theta(\Gamma), e_{\underline{\theta}}(\cdot) \in \mathscr{H}(\Gamma)$. In particular $\operatorname{dim} \mathscr{H}(\Gamma)$ $\geq|\Theta(\Gamma)|$.

Let $J \in \mathbf{J}(\Gamma)$; since $J$ is a "basis" it determines a unique $\underline{\theta} \in \Theta(\Gamma)$ for which $\underline{\theta} \cdot \underline{x}_{\gamma}-\lambda_{\gamma}=0, \forall \gamma \in J$. Moreover, every $\underline{\theta} \in \Theta(\Gamma)$ can be obtained in this way because $\left\langle\Gamma_{\underline{\theta}}\right\rangle=\mathbb{R}^{s}$ and thus contains some $J \in \mathbf{J}(\Gamma)$. Hence we conclude

$$
|\mathbf{J}(\Gamma)| \geq|\Theta(\Gamma)| .
$$

Equality in (2.4) does not hold in general. One may have several $J$ 's which correspond to the same $\underline{\theta} \in \Theta(\Gamma)$. E.g., in case $\underline{\lambda}_{\Gamma}=\underline{0}$ the only node in $\Theta(\Gamma)$ is $\underline{0}$. In order that equality holds in (2.4), it is necessary and sufficient that for each $\underline{\theta} \in \Theta(\Gamma)$ the set $\Gamma_{\underline{\theta}}$ will contain exactly $s$ elements (namely $\Gamma_{\underline{\theta}}$ will be a "basis" from $\mathbf{J}(\Gamma)$ ).

DEFINITION 2.1. We say that $\Gamma$ is a simple defining set if $|\mathbf{J}(\Gamma)|=|\Theta(\Gamma)|$.

In a simple case Proposition 2.1 can be restated as follows.

\section{Proposition 2.2. Assume $\Gamma$ is simple. Then $\operatorname{dim} \mathscr{H}(\Gamma) \geq|\mathbf{J}(\Gamma)|$.}

In order to extend this last result to general defining sets, we need to know how often one encounters simple defining sets. This question was answered in $\left[\mathbf{R}_{1}\right]$ in the following way.

PROPOSITION 2.3. Let $X$ be a set of $n$ nontrivial vectors in $\mathbb{R}^{s}$. Assume $\langle X\rangle=$ $\mathbb{R}^{s}$. For each $\underline{\lambda} \in \mathbb{C}^{n}$, denote by $\Gamma_{\underline{\lambda}}$ the defining set composed of $(X, \underline{\lambda})$. Then the set $\left\{\underline{\lambda} \in \mathbb{C}^{n} \mid \Gamma_{\underline{\lambda}}\right.$ is simple $\}$ is open and dense in $\mathbb{C}^{n}$. Its complement is the union of finitely many hyperplanes of $\mathbb{C}^{n}$.

Now, we combine Theorem 1.2 together with Propositions 2.2 and 2.3 to derive

THEOREM 2.1. For every defining set $\Gamma \operatorname{dim} \mathscr{H}(\Gamma) \geq|\mathbf{J}(\Gamma)|$.

ProOF. Let $\Gamma$ be fixed. Proposition 2.3 ensures the existence of simple defining sets $\left\{\Gamma_{k}\right\}_{k=1}^{\infty}$ such that $X_{\Gamma_{k}}=X_{\Gamma}$ for all $k$ and $\underline{\lambda}_{\Gamma_{k}} \underset{k \rightarrow \infty}{\rightarrow} \underline{\lambda}_{\Gamma}$. By Proposition 2.2

$$
\operatorname{dim} \mathscr{H}\left(\Gamma_{k}\right) \geq\left|\mathbf{J}\left(\Gamma_{k}\right)\right|=|\mathbf{J}(\Gamma)|,
$$

for all $k$. Also since $\left\{X_{\Gamma_{k}}\right\}_{k=1}^{\infty}$ are all equal and $\left\{\underline{\lambda}_{\Gamma_{k}}\right\}_{k=1}^{\infty}$ is convergent, it follows that $\bigcup_{k=1}^{\infty} \Theta\left(\Gamma_{k}\right)$ is bounded, say by $L>0$. Now if $f_{k} \in \mathscr{H}\left(\Gamma_{k}\right)$ and $f_{k} \underset{k \rightarrow \infty}{\rightarrow} f$ in $C^{\infty}\left(\mathbb{R}^{s}\right)$ topology then for every $K \in \mathscr{K}(\Gamma)$

$$
D^{K} f=\lim _{k \rightarrow \infty} D^{K_{k}} f_{k}=0
$$

where $\left\{K_{k}\right\}_{k=1}^{\infty}$ are the corresponding sets in $\left\{\mathscr{K}\left(\Gamma_{k}\right)\right\}_{k=1}^{\infty}$, and thus $f \in \mathscr{H}(\Gamma)$. Application of Theorem 1.2 with $n$ replaced by $|\mathbf{J}(\Gamma)|$ completes the proof.

We now turn to the proof of Theorem 1.2. This proof is primarily based on the following lemma. 
LEMMA 2.1. For each $k \in \mathbb{N}, L>0$ and compact $M \subset \mathbb{R}^{s}$ there exists a constant $c=c(k, L, M)$ such that if $f \in E(k, L)$ and

$$
\left|D^{(\underline{\alpha})} f(\underline{0})\right| \leq \varepsilon, \quad 0 \leq|\underline{\alpha}|<k,
$$

then

$$
|f(\underline{x})| \leq \varepsilon c, \quad \forall \underline{x} \in M .
$$

Proof. Clearly we can and shall assume $\varepsilon=1$. Define $R=R(M)=$ $\sup _{\underline{x} \in M}\|\underline{x}\|_{\infty}$. Fix $\underline{x}^{0} \in M$ and choose $\underline{y} \in \mathbb{R}^{s}$ and $0 \leq t_{0}<R$ such that $\|\underline{y}\|_{\infty}=1$ and $\underline{x}^{0}=\underline{y} t_{0}$. Define

$$
\varphi(t)=f(t \underline{y})
$$

It follows from (2.5) that

$$
\left|\varphi^{(j)}(\underline{0})\right|=\left|(D \underline{y})^{j} f(\underline{0})\right| \leq s^{j} \leq s^{k}, \quad j=0, \ldots, k-1 .
$$

Also, since $f \in E(k, L)$ we have that $\varphi(t)=\sum_{j=1}^{k} c_{j} e_{\lambda_{j}}(t)$ where

$$
\left|\lambda_{j}\right|=\left|\underline{\theta}^{j} \cdot \underline{y}\right| \leq s L \text {. }
$$

Therefore, $\varphi(t)$ lies in the kernel of

$$
\prod_{j=1}^{k}\left(D-\lambda_{j}\right)=D^{k}+a_{k-1} D^{k-1}+\cdots+a_{0}
$$

where $D=d / d t$ and the coefficients $a_{0}, \ldots, a_{k-1}$ satisfy

$$
\left|a_{j}\right|<L_{1}:=2^{k}(1+s L)^{k} .
$$

Writing this differential operator in the usual matrix form we have

$$
\left(\begin{array}{c}
\varphi^{\prime} \\
\varphi^{(2)} \\
\vdots \\
\varphi^{(k)}
\end{array}\right)=\left(\begin{array}{ccccc}
0 & 1 & 0 & \ldots & 0 \\
0 & 0 & 1 & \ldots & 0 \\
\vdots & & & & \vdots \\
0 & . & . & \ldots & 1 \\
-a_{0} & . & . & \ldots & -a_{k-1}
\end{array}\right)\left(\begin{array}{c}
\varphi \\
\varphi^{\prime} \\
\vdots \\
\varphi^{(k-1)}
\end{array}\right)
$$

Thus denoting the matrix in (2.10) by $A$ we find that

$$
\varphi\left(t_{0}\right)=(1,0, \ldots, 0) e^{A t_{0}}\left(\begin{array}{c}
\varphi(0) \\
\varphi^{\prime}(0) \\
\vdots \\
\varphi^{(k-1)}(0)
\end{array}\right) .
$$

From (2.9) we infer that $\left\|e^{A t_{0}}\right\|_{\infty} \leq e^{k L_{1} t_{0}}$, so (2.7) together with (2.11) yields

$$
\left|f\left(\underline{x}^{0}\right)\right|=\left|\varphi\left(t_{0}\right)\right| \leq k s^{k} e^{k L_{1} t_{0}} \leq k s^{k} e^{k L_{1} R} .
$$

Since $\underline{x}^{0} \in M$ was arbitrary the proof is completed by setting

$$
c=k s^{k} e^{\left[k 2^{k}(1+s L)^{k}\right] R(M)} .
$$

The following result easily derived from Lemma 2.1 will be the crux in the proof of Theorem 1.2. 
COROLlaRY 2.1. Let $\left\{f_{j}(\underline{x})\right\}_{j=1}^{\infty} \subset E(n, L)$. Assume

$$
D^{(\underline{\alpha})} f_{j}(\underline{0}) \underset{j \rightarrow \infty}{\rightarrow} a_{\underline{\alpha}} \in \mathbb{C}, \quad 0 \leq|\underline{\alpha}|<2 n .
$$

Then $\left\{f_{j}\right\}_{j=1}^{\infty}$ is convergent in the $C^{\infty}\left(\mathbb{R}^{s}\right)$ topology.

ProOF. First we identify $\mathbb{C}^{s}$ with $\mathbb{R}^{2 s}$ in the usual way

$$
\left(z_{1}, \ldots, z_{s}\right) \sim\left(x_{1}+i x_{s+1}, \ldots, x_{s}+i x_{2 s}\right) .
$$

Every $f \in E(n, L)$ extends to an entire function which we denote by $\tilde{f}$. Then

$$
\frac{\partial \tilde{f}}{\partial z_{j}}=-i \frac{\partial \tilde{f}}{\partial x_{j+s}}=\frac{\partial \tilde{f}}{\partial x_{j}}=\frac{\partial f}{\partial x_{j}}, \quad j=1, \ldots, s .
$$

By $(2.12)$ we know that $\left\{D^{(\underline{\alpha})} f_{j}(\underline{0})\right\}_{j=1}^{\infty}$ is a Cauchy sequence for every $0 \leq|\underline{\alpha}|<$ $2 n$. From (2.13) we conclude that $\left\{D^{(\underline{\alpha})} \tilde{f}(\underline{0})\right\}, 0 \leq|\underline{\alpha}|<2 n$, is also a Cauchy sequence (namely when the derivatives may be taken with respect to all variables $\left(x_{1}, \ldots, x_{2 s}\right)$ ). Thus by application of Lemma 2.1 to $\tilde{f}_{j}-\tilde{f}_{m}$ (with $s$ replaced by $2 s$ and $k=2 n$ ), we find that for every compact $M \subset \mathbb{C}^{s}$ and $\varepsilon>0$ there exists $j_{0}$ such that for every $j, m>j_{0}$ and $\underline{x} \in M$

$$
\left|\tilde{f}_{j}(\underline{x})-\tilde{f}_{m}(\underline{x})\right|<\varepsilon .
$$

Hence $\left\{\tilde{f}_{j}\right\}_{j=1}^{\infty}$ is a Cauchy sequence in $H\left(\mathbb{C}^{s}\right)$ and therefore $\left\{f_{j}\right\}_{j=1}^{\infty}$ is a Cauchy sequence in $C^{\infty}\left(\mathbb{R}^{s}\right)$. Now the corollary follows from the completeness of $C^{\infty}\left(\mathbb{R}^{s}\right)$.

PROOF OF THEOREM 1.2 Let $m=\left(\begin{array}{c}2 n+s-1 \\ s\end{array}\right)$ be the number of all $\underline{\alpha} \in \mathbb{Z}_{+}^{s}$ with $|\underline{\alpha}|<2 n$. Let $\chi$ be a map from $E(n, L)$ to $\mathbb{C}^{m}$ defined as follows:

$$
\chi(f)=\left\{D^{(\underline{\alpha})} f(\underline{0})\right\}_{0 \leq|\underline{\alpha}|<2 n} .
$$

From Lemma 2.1 we conclude that $\chi$ is $1-1$ on each $H_{j}$; hence $\chi\left(H_{j}\right)$ is a subspace of $\mathbb{C}^{m}$ of dimension $\geq d$. So we can choose for each $j$ a set $\left\{f_{j, 1}, \ldots, f_{j, d}\right\} \subset H_{j}$ such that $\left\{\chi\left(f_{j, k}\right)\right\}_{k=1}^{d}$ is an orthonormal set in $\mathbb{C}^{m}$. Since the unit sphere of $\mathbb{C}^{m}$ is compact we can find a subsequence $\left\{j_{r}\right\}_{r=1}^{\infty}$ such that $\left\{\chi\left(f_{j_{r}, k}\right)\right\}_{r=1}^{\infty}$ is convergent for $k=1, \ldots, d$. By Corollary 2.1 there exist $f_{1}, \ldots, f_{d}$ in $C^{\infty}\left(\mathbf{R}^{s}\right)$ such that

$$
f_{j_{r}, k} \underset{r \rightarrow \infty}{\rightarrow} f_{k}, \quad k=1, \ldots, d,
$$

in $\mathbb{C}^{\infty}\left(\mathbb{R}^{s}\right)$. Denote $H=\left\langle f_{1}, \ldots, f_{d}\right\rangle$. Let us show that $\operatorname{dim} H=d$ by proving that $f_{1}, \ldots, f_{d}$ are linearly independent. Assume $\underline{a} \in \mathbb{C}^{d}, \sum_{k=1}^{d} a_{k} f_{k} \equiv 0$ on $\mathbb{R}^{s}$. Then

$$
\lim _{r \rightarrow \infty} \sum_{k=1}^{d} a_{k} f_{j_{r}, k}=0
$$

in $C^{\infty}\left(\mathbb{R}^{s}\right)$ topology. Since $\chi$ is continuous on $C^{\infty}\left(\mathbb{R}^{s}\right)$ we conclude that

$$
\lim _{r \rightarrow \infty} \sum_{k=1}^{d} a_{k} \chi\left(f_{j_{r}, k}\right)=0
$$

in $\mathbb{C}^{m}$. But for each $r\left\{\chi\left(f_{j_{r}, k}\right)\right\}_{k=1}^{d}$ is orthonormal and therefore

$$
\sum_{k=1}^{d}\left|a_{k}\right|^{2}=\left\|\sum_{k=1}^{d} a_{k} \chi\left(f_{j_{r}, k}\right)\right\|_{2} \underset{r \rightarrow \infty}{\rightarrow} 0 .
$$

Consequently $\underline{a}=\underline{0}$ and the proof of Theorem 1.2 is completed. 
3. Proof of Theorem 1.3. Before proving Theorem 1.3 we state and prove an apparently weaker result.

LEMMA 3.1. (a) Every finite dimensional $D$-invariant subspace $H$ of a $P E$ space is also a PE space.

(b) Every finite dimensional shift-invariant subspace $S$ of a PE sequence space is also a PE space.

Proof. (a) Let $f \in H$. Since $f$ belongs to a $P E$ space it has the form

$$
f(\underline{x})=\sum_{j=1}^{n} p_{j}(\underline{x}) e_{\underline{\theta}^{j}}(\underline{x}), \quad p_{j} \in \pi, \underline{\theta}^{j} \in \mathbb{C}^{s}, j=1, \ldots, n .
$$

Without loss of generality we can assume that $\left\{\underline{\theta}^{j}\right\}_{j=1}^{n}$ are pairwise distinct. Thus, for $j=2, \ldots, n$ there exist $\underline{x}^{j} \in \mathbb{R}^{s}$ and $\lambda_{j} \in \mathbb{C}$ such that

$$
\underline{x}^{j} \cdot \underline{\theta}^{1}-\lambda_{j} \neq 0, \quad \underline{x}^{j} \cdot \underline{\theta}^{j}-\lambda_{j}=0 .
$$

Denote

$$
D^{j}=D_{\underline{x}^{j}}-\lambda_{j}
$$

then for every $p \in \pi$

$$
D^{j}\left[e_{\underline{\theta}^{1}}(\cdot) p(\cdot)\right]=e_{\underline{\theta}^{1}}(\cdot) q(\cdot), \quad \operatorname{deg} q(\cdot)=\operatorname{deg} p(\cdot),
$$

but

$$
D^{j}\left[e_{\underline{\theta}^{j}}(\cdot) p(\cdot)\right]=e_{\underline{\theta}^{j}}(\cdot)\left[D_{\underline{x}^{j}} p(\cdot)\right] .
$$

Defining $L_{1}=\prod_{j=2}^{n}\left(D^{j}\right)^{\operatorname{deg} p_{j}+1}$, we see that $L_{1} f(\cdot)=e_{\underline{\theta}^{1}}(\cdot) q(\cdot)$ where $\operatorname{deg} q(\cdot)=$ $\operatorname{deg} p_{1}(\cdot)$. Since $H$ is $D$-invariant, we know that $L_{1} f \in H$. Denote by $H_{1}$ the subspace of $H$ consisting of elements of the form $e_{\underline{\theta}^{1}}(\cdot) p(\cdot), p \in \pi$. Since $H_{1}$ is of finite dimension, the preceding arguments show that $L_{1}$ is an injective map of $H_{1}$ onto itself. Thus, since $q(\cdot) e_{\theta^{1}}(\cdot) \in H_{1}$ and $L_{1}\left(e_{\underline{\theta}^{1}}(\cdot) p_{1}(\cdot)\right)=e_{\underline{\theta}^{1}}(\cdot) q(\cdot)$ we must have $e_{\underline{\theta}^{1}}(\cdot) p_{1}(\cdot) \in H_{1} \subset H$. This argument can be applied to any index other than 1 ; consequently $\left\{e_{\theta^{j}}(\cdot) p_{j}(\cdot)\right\}_{j=1}^{n} \subset H$ and (a) is established.

The proof of (b) is analogous: if $u \in S$, then since $S$ is included in a $P E$ space, we have

$$
u(\underline{\alpha})=\sum_{j=1}^{n} e_{\underline{\theta}^{j}}(\underline{\alpha}) p_{j}(\alpha), \quad p_{j} \in \pi, \underline{\theta}^{j} \in \mathbb{C}^{s}, j=1, \ldots, n .
$$

Since we take $\underline{\alpha} \in \mathbb{Z}^{s}$ we can assume $\underline{\theta}^{j}-\underline{\theta}^{k} \notin 2 \pi i \mathbb{Z}^{s}$ for $j \neq k$. Given $2 \leq j \leq n$ we can therefore find $\underline{\alpha}^{j} \in \mathbb{Z}^{s}$ and $\lambda_{j} \in \mathbb{C}$ such that

$$
e^{\underline{\alpha}^{j} \cdot \underline{\theta}^{1}-\lambda_{j}} \neq 1, \quad e^{\underline{\alpha}^{j} \cdot \underline{\theta}^{j}-\lambda_{j}}=1,
$$

and denote

$$
\Delta_{1}=\prod_{j=2}^{n}\left(E_{\underline{\alpha}^{j}}-e^{\lambda_{j}}\right)^{\operatorname{deg} p_{j}+1} .
$$

The rest of the proof is identical to that of part (a) with $\Delta_{1}$ replacing $L_{1}$.

Next, we reduce the first part of Theorem 1.3 from $\mathscr{D}^{\prime}\left(\mathbb{R}^{s}\right)$ to $C^{\infty}\left(\mathbb{R}^{s}\right)$ : 
THEOREM 3.2. Let $H$ be a subspace of $\mathscr{D}^{\prime}\left(\mathbb{R}^{s}\right)$ which is closed under convolution with compactly supported $C^{\infty}$ functions. Assume $H \cap C^{\infty}\left(\mathbb{R}^{s}\right)$ is finite dimensional. Then $H \subset C^{\infty}\left(\mathbb{R}^{s}\right)$.

ProOF. Denote $N=\operatorname{dim}\left(H \cap C^{\infty}\left(\mathbb{R}^{s}\right)\right)$. Let $f_{1}, \ldots, f_{N+1} \subset H$. Let $\left\{\varphi_{h}\right\}_{h>0}$ be an infinitely differentiable approximate identity of compact support (see $[\mathbf{R u}$, p. 157]). Then $\left\{f_{j} * \varphi_{h}\right\}_{j=1}^{N+1} \subset H \cap C^{\infty}\left(\mathbb{R}^{s}\right)$, so there exist $\underline{c}_{h}:=\left\{c_{h, j}\right\}_{j=1}^{N+1}$ such that

$$
\left(\sum_{j=1}^{N+1} c_{h, j} f_{j}\right) * \varphi_{h}=\sum_{j=1}^{N+1} c_{h, j}\left(f_{j} * \varphi_{h}\right) \equiv 0 .
$$

We can assume $\sum_{j=1}^{N+1} c_{h, j}^{2}=1$ for all $h$ and the compactness of the unit ball in $\mathbb{C}^{N+1}$ implies the existence of a sequence $h_{k} \underset{k \rightarrow \infty}{\rightarrow} 0$ such that $\underline{c}_{h_{k}} \underset{k \rightarrow \infty}{\rightarrow} \underline{c}^{\circ} \neq 0$. Therefore

$$
\sum_{j=1}^{N+1} c_{j}^{\circ} f_{j}=\lim _{k \rightarrow \infty}\left(\sum_{j=1}^{N+1} c_{h_{k}, j} f_{j}\right) * \varphi_{h_{k}}=0 .
$$

Consequently, $\operatorname{dim} H \leq N$ which implies $H=H \cap C^{\infty}\left(\mathbb{R}^{s}\right)$.

We now turn to the proof of Theorem 1.3(a). Denote the space under consideration by $H$. Let $\left\{\underline{e}^{j}\right\}_{j=1}^{s}$ be the standard basis of $\mathbb{R}^{s}$. We know that $D_{\underline{e}^{j}}$ is a linear transformation which maps $H$ into itself. Denote by $\left.D_{\underline{e}^{j}}\right|_{H}$ the restriction of $D_{\underline{e}^{j}}$ to $H$ and by $\left(\lambda_{j, 1}, \ldots, \lambda_{j, n}\right)$ the spectrum of $\left.D_{\underline{e}^{j}}\right|_{H}$ counting (algebraic) multiplicities. Then by the Cayley-Hamilton theorem

$$
D^{j}:=\prod_{k=1}^{n}\left(\left.D_{\underline{e}^{j}}\right|_{H}-\lambda_{j, k}\right)=0, \quad H \subset \bigcap_{j=1}^{s} \operatorname{ker} D^{j}=: H_{1} .
$$

Clearly, $H_{1} \cap C^{\infty}\left(\mathbb{R}^{s}\right)$ is a finite dimensional $P E$ space. In fact a basis to $H_{1} \cap$ $C^{\infty}\left(\mathbb{R}^{s}\right)$ is given in terms of the functions $e_{\underline{\lambda}}(\underline{x}) \underline{x} \underline{\underline{\nu}}$, where for every $1 \leq j \leq s, \lambda_{j}$ lies in the spectrum of $D_{\underline{e}^{j}} \mid H$ and $\nu_{j}-1$ does not exceed the multiplicity of $\lambda_{j}$.

But $H_{1}$ is also closed under convolution with test functions. Indeed for $f \in H_{1}$ and $g \in \mathscr{D}\left(\mathbb{R}^{s}\right)$ one has

$$
D^{j}(f * g)=\left(D^{j} f\right) * g=0, \quad j=1, \ldots, s .
$$

Therefore, application of Lemma 3.2 yields that $H_{1}$ is a $P E$ space and hence by Lemma 3.1 so is $H$. This completes the proof of Theorem 1.3(a).

To prove (b), note that the same arguments used in the proof of part (a) show the existence of $\underline{\lambda}^{1}, \ldots, \underline{\lambda}^{s} \in \mathbb{C}^{n}$ such that

$$
S \subset \bigcap_{j=1}^{s}\left(\operatorname{ker} \prod_{k=1}^{n}\left(E_{\underline{e}^{j}}-\lambda_{k}^{j}\right)\right)=: S_{1} .
$$

Since $S_{1}$ is clearly a $P E$ sequence space, Lemma 3.1(b) yields the desired result.

In the analysis of $\mathscr{H}(\Gamma)$ and $\mathscr{S}_{h}(\Gamma)$ we frequently investigate the restriction of a $P E$ space to the lattice points. When doing so a certain precaution is needed: in case $\underline{\theta}^{1}-\underline{\theta^{2}} \in 2 \pi i \mathbb{Z}^{s}$ one has

$$
\left.\left.e_{\underline{\theta}^{1}}(\cdot)\right|_{\mathbb{Z}^{s}} \equiv e_{\underline{\theta}^{2}}(\cdot)\right|_{\mathbb{Z}^{s}}
$$

and therefore a function cannot be identified with its restriction. The following proposition shows that besides this case restriction to $\mathbb{Z}^{s}$ is "safe": 
Proposition 3.1. Let $\left\{\underline{\theta}^{j}\right\}_{j=1}^{n} \subset \mathbb{C}^{s}, \underline{\theta}^{j}-\underline{\theta}^{k} \notin 2 \pi i \mathbb{Z}^{s}$ for $j \neq k$. Let

$$
f_{j}(\cdot)=e_{\underline{\theta}^{j}}(\cdot) p_{j}(\cdot) \quad p_{j} \in \pi, j=1, \ldots, n .
$$

Then $\left\{f_{j}(\cdot)\right\}_{j=1}^{n}$ are linearly independent on $\mathbb{Z}^{s}$.

Proof. Assume $\sum_{j=1}^{n} f_{j}(\underline{\alpha})=0, \forall \underline{\alpha} \subset \mathbb{Z}^{s}$. Since $\underline{\theta}^{k}-\underline{\theta}^{j} \notin 2 \pi i \mathbb{Z}^{s}$ for $k \neq j$, one can construct as in the proof of Lemma 3.1(b) a difference operator $\Delta_{1}$ such that

$$
\Delta_{1} f_{j}(\cdot) \equiv 0, \quad j=2, \ldots, n, \quad \Delta_{1} f_{1}(\cdot)=e_{\underline{\theta}^{1}}(\cdot) q(\cdot),
$$

where $\operatorname{deg} q=\operatorname{deg} p_{1}$. Since $\sum_{j=1}^{n} f_{j}$ vanishes on $\mathbb{Z}^{s}$ we conclude that $q(\cdot)$ vanishes on $\mathbb{Z}^{s}$. Thus $q(\cdot) \equiv 0$ and hence $p_{1}(\cdot)=0$. Application of induction to $n$ completes the proof.

Note that every $\left.e_{\underline{\theta}^{j}}(\cdot)\right|_{\mathbb{Z}^{s}}$ can be viewed as a complex homomorphism of $\mathbb{Z}^{s}$. Thus the case $p_{j}=$ const $_{j}, j=1, \ldots, n$, could also be obtained by Artin's theorem about the linear independence of homomorphisms (see, e.g., [L, p. 209]).

Finally, we mention the following fact which is easily verified by the methods used in Lemma 3.1.

COROLLARY 3.1. Let $F$ be a $D$-invariant subspace of a $P E$ distribution space or a shift invariant subspace of a PE sequence space. Then $F$ contains an exponential.

4. The structure of $\mathscr{H}(\Gamma)$ and $\mathscr{S}_{h}(\Gamma)$. We first show that $\mathscr{S}_{h}(\Gamma)$ and $\mathscr{H}(\Gamma)$ are of finite dimension, which allows us to deduce from Theorem 1.3 that they are $P E$ spaces. This fact greatly simplifies the analysis of their structure.

Fix $h>0$ and $J \in \mathbf{J}(\Gamma)$ and denote

$$
G_{h}^{J}=\left\{X_{J} \underline{\alpha} \mid \underline{\alpha} \in \mathbb{Z}_{h}^{s}\right\} .
$$

Since $X_{J} \subset \mathbb{Z}^{s}$ we know that $G_{h}^{J}$ is a subgroup of $\mathbb{Z}_{h}^{s}$ of index $\left|\operatorname{det} X_{J}\right|$. On the other hand, it is easily see that the sets $\left\{R_{h}(J)-\underline{\alpha} \mid \underline{\alpha} \in G_{h}^{J}\right\}$ form a partition of $\mathbb{R}^{s}$. This means that for every $\underline{y} \in A_{h}(\Gamma)$, each coset of $G_{h}^{J}$ is uniquely represented in $b_{h}^{J}(\underline{y})$. In particular

$$
\left|b_{h}^{J}(\underline{y})\right|=\left|\operatorname{det} X_{J}\right|, \quad \forall \underline{y} \in A_{h}(\Gamma) .
$$

(4.2) is actually a special case of $\left[\mathrm{DM}_{2}\right.$, Theorem 3.1] which will be used in its full generality in the next section.

THEOREM 4.1. Let $\underline{y} \in A_{h}(\Gamma)$. Then the operation of restricting elements of $\mathscr{S}_{h}(\Gamma)$ from $\mathbb{Z}_{h}^{s}$ to $b_{h}^{\Gamma}(\underline{y})$ is injective. In particular $\operatorname{dim} \mathscr{S}_{h}(\Gamma) \leq\left|b{ }_{h}^{\Gamma}(\underline{y})\right|$.

PrOOF. We prove the theorem by induction on $|\Gamma| \geq s$. Assume $|\Gamma|=s$ (and $\left.\langle\Gamma\rangle=\mathbb{R}^{s}\right)$. Let $u(\cdot) \in \mathscr{S}_{h}(\Gamma)$ and assume that $\left.u\right|_{b_{h}^{\Gamma}(\underline{y})} \equiv 0$. Since $|\Gamma|=s$ we have $\langle\Gamma \backslash \gamma\rangle \neq \mathbb{R}^{s}$ for all $\gamma \in \Gamma$ and therefore

$$
u(\cdot) \in \bigcap_{\gamma \in \Gamma} \operatorname{ker} \nabla_{h}^{\gamma} .
$$

Using (4.3) it is easily seen that if $u\left(\underline{\alpha}^{0}\right)=0$ for some $\underline{\alpha}^{0} \in \mathbb{Z}_{h}^{s}$ then $u(\cdot)$ vanishes on the coset $\underline{\alpha}^{0}+G_{h}^{\Gamma}$. Since $b_{h}^{\Gamma}(\underline{y})$ intersects every coset of $G_{h}^{\Gamma}$ in $\mathbb{Z}_{h}^{s}$ we conclude $u \equiv 0$. 
Assume $|\Gamma|>s, u \in \mathscr{S}(\Gamma),\left.u\right|_{b_{h}^{\Gamma}(\underline{y})} \equiv 0$. Let $\gamma \in \Gamma$. If $\langle\Gamma \backslash \gamma\rangle \neq \mathbb{R}^{s}$ then $\nabla_{h}^{\gamma} u \equiv 0$. If $\langle\Gamma \backslash \gamma\rangle=\mathbb{R}^{s}$ then we claim that

$$
\left.\nabla_{h}^{\gamma} u\right|_{b_{h}^{\Gamma \backslash \gamma}(\underline{y})} \equiv 0 .
$$

To see this assume $\underline{\alpha} \in b_{h}^{\Gamma \backslash \gamma}(\underline{y})$; then it follows from (1.4) and (1.12) that $\underline{\alpha}$, $\underline{\alpha}-h \underline{x}_{\gamma} \in b_{h}^{\Gamma}(\underline{y})$. Therefore $\nabla_{h}^{\gamma} u(\underline{\alpha})=0-0=0$.

By the induction hypothesis (4.4) implies $\nabla_{h}^{\gamma} u \equiv 0$. This implies that $\nabla_{h}^{\gamma} u=0$ for all $\gamma \in \Gamma$. Let $J \in \mathbf{J}(\Gamma)$. Then $\left.u\right|_{b_{h}^{J}(\underline{y})} \equiv 0$ because $b_{h}^{J}(\underline{y}) \subset b_{h}^{\Gamma}(\underline{y})$. Hence by the first part of the proof we conclude that $u \equiv 0$.

REMARK 4.1. It is clear that $\min \left\{\left|b_{h}^{\Gamma}(y)\right| \mid y \in A_{h}(\Gamma)\right\}$ is independent of $h$. Therefore we see that the bound obtained in Theorem 4.1 to $\operatorname{dim} \mathscr{S}_{h}(\Gamma)$ is also independent of $h$.

REMARK 4.2. Comparison of Theorem 4.1 here with Theorem 3.3 of $\left[\mathbf{D M}_{3}\right]$ shows that Theorem 3.2 in $\left[\mathbf{D M}_{3}\right]$ is actually redundant.

We apply now Theorem 1.3 to show that $\mathscr{S}_{h}(\Gamma)$ and $\mathscr{H}(\Gamma)$ are $P E$ spaces. Note that Theorem 1.3(b) was proved only with respect to sequences defined on $\mathbb{Z}^{s}$ rather than $\mathbb{Z}_{h}^{s}$. Yet, the extension to arbitrary $h$ is trivial.

COROLlaRY 4.1. $\mathscr{H}(\Gamma)$ and $\mathscr{S}_{h}(\Gamma)$ are finite dimensional PE spaces.

PROOF. In view of Theorem 1.3 and the obvious fact that $\mathscr{H}(\Gamma)$ is $D$-invariant and $\mathscr{S}_{h}(\Gamma)$ is shift invariant, it is enough to prove that these spaces are of finite dimension. For $\mathscr{S}_{h}(\Gamma)$ this was proved in Theorem 4.1.

To prove that $\mathscr{H}(\Gamma)$ is of finite dimension, let $N \in \mathbb{N}$ and assume that $\left\{f_{j}(\cdot)\right\}_{j=1}^{N}$ are linearly independent functions in $\mathscr{H}(\Gamma) \cap C^{\infty}\left(\mathbb{R}^{s}\right)$. Choose $h>0$ small enough such that

$$
u_{j}(\cdot):=\left.f_{j}(\cdot)\right|_{\mathbb{Z}_{h}^{s}}, \quad j=1, \ldots, N
$$

are linearly independent on $\mathbb{Z}_{h}^{s}$. From (1.11) one easily deduces $u_{j} \in \mathscr{S}_{h}(\Gamma), j=$ $1, \ldots, N$. Thus since $\operatorname{dim} \mathscr{S}_{h}(\Gamma)$ is bounded uniformly in $h$, we see that $\mathscr{H}(\Gamma) \cap$ $C^{\infty}\left(\mathbb{R}^{s}\right)$ is of finite dimension. Application of Lemma 3.2 completes the proof.

REMARK 4.3. Theorem 4.1 admits a continuous analog which shows in particular that $\operatorname{dim} \mathscr{H}(\Gamma) \leq|\mathbf{J}(\Gamma)|$ (see [DR]). That result can be used to give an alternative proof for Corollary 4.1, hence to derive Theorem 1.1 regardless of the restriction $X_{\Gamma} \subset \mathbb{Z}^{s}$.

Now, we use Corollary 4.1 to reveal the structure of $\mathscr{H}(\Gamma)$. For this purpose let $\Gamma_{\underline{\theta}}$ and $\Theta(\Gamma)$ be as in (2.2), (2.3) and denote for every $\underline{\theta} \in \Theta(\Gamma)$

$$
X_{\underline{\theta}}=\left\{(\underline{x}, 0) \mid \underline{x} \in X_{\Gamma_{\underline{\theta}}}\right\},
$$

i.e., $X_{\underline{\theta}}$ is the defining set obtained from $\Gamma_{\underline{\theta}}$ when replacing $\underline{\lambda}_{\Gamma_{\underline{\theta}}}$ by $\underline{0}$.

THEOREM 4.2. The space $\mathscr{H}(\Gamma)$ admits the following direct sum decomposition

$$
\mathscr{H}(\Gamma)=\bigoplus_{\underline{\theta} \in \Theta(\Gamma)} \mathscr{H}\left(\Gamma_{\underline{\theta}}\right)=\bigoplus_{\underline{\theta} \in \Theta(\Gamma)} e_{\underline{\theta}}(\cdot) \mathscr{H}\left(X_{\underline{\theta}}\right),
$$

where each $\mathscr{H}\left(X_{\underline{\theta}}\right)$ is a nontrivial polynomial space. In particular

$$
e_{\underline{\theta}}(\cdot) \in \mathscr{H}(\Gamma) \text { if and only if } \underline{\theta} \in \Theta(\Gamma) .
$$


PROOF. By Corollary 4.1 it is enough to identify in $\mathscr{H}(\Gamma)$ the functions of the form $e_{\underline{\theta}}(\cdot) p(\cdot), p \in \pi, \underline{\theta} \in \mathbb{C}^{s}$. We start by proving that $e_{\underline{\theta}}(\cdot) p(\cdot) \in \mathscr{H}(\Gamma)$ only if $\underline{\theta} \in \Theta(\bar{\Gamma})$. Given such a function we see that for $\gamma \in \Gamma_{\underline{\theta}}$

$$
D^{\gamma}\left(e_{\underline{\theta}}(\cdot) p(\cdot)\right)=e_{\underline{\theta}}(\cdot) D_{\underline{x}_{\gamma}} p(\cdot),
$$

while for $\gamma \in \Gamma \backslash \Gamma_{\underline{\theta}}$

$$
D^{\gamma}\left(e_{\underline{\theta}}(\cdot) p(\cdot)\right)=e_{\underline{\theta}}(\cdot) q(\cdot), \quad \text { where } \operatorname{deg} q=\operatorname{deg} p .
$$

Thus for $K \subset \Gamma$

$$
D^{K}\left(e_{\underline{\theta}}(\cdot) p(\cdot)\right) \equiv 0 \Leftrightarrow D^{K \cap \Gamma_{\underline{\theta}}}\left(e_{\underline{\theta}}(\cdot) p(\cdot)\right) \equiv 0 \Leftrightarrow D^{X_{\Gamma_{\underline{\theta}} \cap K}} p(\cdot) \equiv 0 .
$$

If $\underline{\theta} \notin \Theta(\Gamma)$ then $\left\langle\Gamma_{\underline{\theta}}\right\rangle \neq \mathbb{R}^{s}$ and hence $\Gamma \backslash \Gamma_{\underline{\theta}} \in \mathscr{K}(\Gamma)$. On the other hand, (4.8) shows that $D^{\Gamma \backslash \Gamma_{\underline{\theta}}}\left(e_{\underline{\theta}}(\cdot) p(\cdot)\right) \neq 0$ because $\left(\Gamma \backslash \Gamma_{\underline{\theta}}\right) \cap \Gamma_{\underline{\theta}}=\varnothing$. Consequently $e_{\underline{\theta}}(\cdot) p(\cdot) \notin$ $\mathscr{H}(\Gamma)$. Combining this result with Proposition 2.1 verifies (4.7), and since $\Theta\left(X_{\underline{\theta}}\right)=$ $\{\underline{0}\}$ for every $\underline{\theta} \in \Theta(\Gamma)$, we also conclude that $\mathscr{H}\left(X_{\underline{\theta}}\right)$ contains only polynomials. Finally, (4.6) easily follows from (4.8) and the observation that

$$
\mathscr{K}\left(\Gamma_{\underline{\theta}}\right)=\left\{K \cap \Gamma_{\underline{\theta}} \mid K \in \mathscr{K}(\Gamma)\right\} .
$$

We now turn to the analysis of $\mathscr{S}_{h}(\Gamma)$. Here for $\underline{\theta} \in \mathbb{C}^{s}$ we define

$$
\tilde{\Gamma}_{\underline{\theta}, h}=\left\{\gamma \in \Gamma \mid e^{h\left(\lambda_{\gamma}-\underline{x}_{\gamma} \cdot \underline{\theta}\right)}=1\right\},
$$

and introduce the set of "discrete nodes"

$$
\tilde{\Theta}_{h}(\Gamma)=\left\{\underline{\theta} \in \mathbb{C}^{s} \mid\left\langle\tilde{\Gamma}_{\underline{\theta}, h}\right\rangle=\mathbb{R}^{s}, \operatorname{Im} \underline{\theta} \in\left[0,2 \pi h^{-1}\right)^{s}\right\} .
$$

To analyze $\mathscr{S}_{h}(\Gamma)$ we first prove the following result.

LEMMA 4.1. Let $K \subset \Gamma, p \in \pi$. Then

$$
\nabla_{h}^{X_{K}} p \equiv 0 \Rightarrow D^{X_{K}} p \equiv 0 .
$$
that

PROOF. Given a compactly supported $\phi \in \mathscr{D}^{\prime}\left(\mathbb{R}^{s}\right)$ and $q \in \pi$ it can be shown

$$
\phi * q=\hat{\phi}(\underline{0}) q(\underline{x})+\text { a polynomial of lower degree than } q(\underline{x}) .
$$

In particular $\phi * q \equiv 0$ and $\hat{\phi}(\underline{0}) \neq 0$ imply $q \equiv 0$. Now assuming $\nabla_{h}^{X_{K}} p \equiv 0$ we get from (1.11) that $B_{h}\left(X_{K}\right) * D^{X_{K}} p \equiv 0$, and since $\hat{B}_{h}\left(X_{K} \mid \underline{0}\right) \neq 0$ then $D^{X_{K}} p \equiv 0$.

Now, we combine Corollary 4.1 together with Lemma 4.1 and Proposition 2.1 to conclude

THEOREM 4.3 .

$$
\mathscr{S}_{h}(\Gamma)=\bigoplus_{\underline{\theta} \in \tilde{\Theta}_{h}(\Gamma)} e_{\underline{\theta}}(\cdot) \mathscr{H}\left(\tilde{X}_{\underline{\theta}, h}\right)
$$

where

$$
\tilde{X}_{\underline{\theta}, h}=\left\{\left(\underline{x}_{\gamma}, 0\right) \mid \gamma \in \tilde{\Gamma}_{\underline{\theta}, h}\right\} .
$$

REMARK 4.4. By the convention stated in the introduction only restrictions to $\mathbb{Z}_{h}^{s}$ are considered in (4.11).

ProOF. The sum $\bigoplus_{\theta \in \tilde{\Theta}_{h}(\Gamma)} e_{\underline{\theta}}(\cdot) \mathscr{H}\left(\tilde{X}_{\underline{\theta}, h}\right)$ is indeed direct. This follows from (4.10), Proposition 3.1 and the fact that each $\mathscr{H}\left(\tilde{X}_{\underline{\theta}, h}\right)$ contains only polynomials. 
Now, for $\underline{\theta} \in \mathbb{C}^{s}, \gamma \in \tilde{\Gamma}_{\underline{\theta}, h}$ and $p \in \pi$

$$
\nabla_{h}^{\gamma}\left(e_{\underline{\theta}}(\cdot) p(\cdot)\right)=e_{\underline{\theta}}(\cdot) \nabla_{h}^{\underline{x}} p(\cdot),
$$

while for $\gamma \notin \tilde{\Gamma}_{\underline{\theta}, h}$

$$
\nabla_{h}^{\gamma}\left(e_{\underline{\theta}}(\cdot) p(\cdot)\right)=e_{\underline{\theta}}(\cdot) q(\cdot) \quad \text { where } \operatorname{deg} p=\operatorname{deg} q .
$$

Utilizing Lemma 4.1 we see that our claim is obtained by the same arguments used in the proof of Theorem 4.2.

Usually the set of discrete nodes $\tilde{\Theta}_{h}(\Gamma)$ is richer than that of nodes $\Theta(\Gamma)$. On the other hand, if $\underline{\theta} \in \tilde{\Theta}_{h}(\Gamma) \cap \Theta(\Gamma)$ we may have $\Gamma_{\underline{\theta}} \varsubsetneqq \tilde{\Gamma}_{\underline{\theta}, h}$. Yet, this last case is not "stable" and can be avoided by a refinement of the mesh.

Proposition 4.1. For every $\Gamma$ there exists a positive number $h_{0}$, which depends only on $X_{\Gamma}$ and $\left\|\underline{\lambda}_{\Gamma}\right\|_{\infty}$, such that for every $h<h_{0}$

(a) $\Theta(\Gamma) \subset \tilde{\Theta}_{h}(\Gamma)$, and

(b) $\Gamma_{\underline{\theta}}=\tilde{\Gamma}_{\underline{\theta}, h}, \forall \underline{\theta} \in \Theta(\Gamma)$.

Moreover, if $\underline{\underline{\lambda}}_{\Gamma} \subset \mathbb{R}$ then (a), (b) hold for every $h>0$.

Proof. It is easy to see that there exists $c_{1}>0$ which depends only on $X_{\Gamma}$ such that

$$
\|\underline{\theta}\|_{\infty} \leq c_{1}\left\|\underline{\lambda}_{\Gamma}\right\|_{\infty}, \quad \forall \underline{\theta} \in \Theta(\Gamma) .
$$

So, in view of (4.10), the choice $h<2 \pi\left(c_{1}\left\|\underline{\lambda}_{\Gamma}\right\|_{\infty}\right)^{-1}$ gives (a). Given $\underline{\theta} \in \mathbb{C}^{s}$ we see from (4.9) that

$$
\gamma \in \tilde{\Gamma}_{\underline{\theta}, h} \Leftrightarrow h\left(\lambda_{\gamma}-\underline{x}_{\gamma} \cdot \underline{\theta}\right) \in 2 \pi i \mathbb{Z}^{s} .
$$

But (4.12) implies the existence of $c_{2} \geq c_{1}$ (dependent only on $X_{\Gamma}$ ) such that $\left|\lambda_{\gamma}-\underline{x}_{\gamma} \cdot \underline{\theta}\right| \leq c_{2}\left\|\underline{\lambda}_{\Gamma}\right\|_{\infty}$ for all $\gamma \in \Gamma, \underline{\theta} \in \Theta(\Gamma)$. Thus given $\underline{\theta} \in \Theta(\Gamma)$ and $\gamma \in \Gamma \backslash \Gamma_{\underline{\theta}}$ we have for $h<h_{0}=2 \pi\left(c_{2}\left\|\underline{\lambda}_{\Gamma}\right\|_{\infty}\right)^{-1}$

$$
0<\left|h\left(\lambda_{\gamma}-\underline{x}_{\gamma} \cdot \underline{\theta}\right)\right|<h c_{2}\left\|\underline{\lambda}_{\gamma}\right\|_{\infty}<2 \pi,
$$

which shows that $\gamma \notin \tilde{\Gamma}_{\underline{\theta}, h}$. Thus $\Gamma_{\underline{\theta}} \supset \tilde{\Gamma}_{\underline{\theta}, h}$ whereas the opposite inclusion is trivial.

Finally, note that if $\underline{\lambda}_{\Gamma} \subset \mathbb{R}$ then clearly $\Theta(\Gamma) \subset \mathbb{R}^{s}$ so that for every $h>0$,

$$
\gamma \in \tilde{\Gamma}_{\underline{\theta}, h} \Leftrightarrow h\left(\lambda_{\gamma}-\underline{x}_{\gamma} \cdot \underline{\theta}\right) \in 2 \pi i \mathbb{Z}^{s} \Leftrightarrow \lambda_{\gamma}-\underline{x}_{\gamma} \cdot \underline{\theta}=0 \Leftrightarrow \gamma \in \Gamma_{\underline{\theta}}
$$

which proves (b), while (a) in this case is trivial.

Note that Proposition 4.1 says that for small enough $h, \mathscr{H}(\Gamma)$ is embedded in $\mathscr{S}_{h}(\Gamma)$. Note also that the proof of this proposition shows that $\tilde{\Theta}_{h}(\Gamma)-\Theta(\Gamma)$ tends to infinity as $h$ tends to zero.

Comparing (4.9) and (1.2) we see that for $\underline{\theta} \in \Theta(\Gamma)$

$$
\tilde{\Gamma}_{\underline{\theta}, h}=\Gamma_{\underline{\theta}} \Leftrightarrow \hat{B}_{h}(\Gamma \mid-i \underline{\theta}) \neq 0 .
$$

Therefore Proposition 4.1 implies the following corollary. 
COROLLARY 4.2. For every $\Gamma$ there exists a positive $h_{0}$, which depends only on $X_{\Gamma}$ and $\left\|\underline{\lambda}_{\Gamma}\right\|_{\infty}$ such that for every $h<h_{0}$

$$
\hat{B}_{h}(\Gamma \mid-i \underline{\theta}) \neq 0, \quad \forall \underline{\theta} \in \Theta(\Gamma) .
$$

In case $\underline{\lambda}_{\Gamma} \subset \mathbb{R},(4.14)$ holds for every $h$.

5. Proof of Theorem 1.1. Here we complete the proof of Theorem 1.1 and establish several additional properties of $\mathscr{H}(\Gamma)$ and $\mathscr{S}_{h}(\Gamma)$.

First we derive the following quantitative result about the set $\tilde{\Theta}_{h}(\Gamma)$ of discrete nodes:

LEMMA 5.1. For any $J \in \mathbf{J}(\Gamma)$

$$
\left|\tilde{\Theta}_{h}(J)\right|=\left|\operatorname{det} X_{J}\right| \text {. }
$$

Proof. Fix $\underline{\theta}^{0} \in \tilde{\Theta}_{h}(J)$ and let $\underline{\theta} \in \mathbb{C}^{s}$ be arbitrary. Then by (4.9), (4.10), $\underline{\theta} \in \tilde{\Theta}_{h}(J)$ if and only if $e^{h\left(\underline{\theta}-\underline{\theta}^{0}\right) \cdot \underline{x}_{\gamma}}=1, \forall \gamma \in J$. This condition is equivalent to $e_{\underline{\theta}-\underline{\theta}^{0}}(\cdot)$ being a complex homomorphism of $\mathbb{Z}_{h}^{s}$ which is constant on the cosets of $\bar{G}_{h}^{J}$ in $\mathbb{Z}_{h}^{s}$. Each such homomorphism can be factored as follows

$$
\begin{array}{ccc}
\mathbb{Z}_{h}^{s} & \vec{q} & \mathbb{Z}_{h}^{s} / G_{h}^{J} \\
e_{\underline{\theta}-\underline{\theta}^{0}} \searrow & & \swarrow \chi \\
& \mathbb{C} &
\end{array}
$$

where $q$ is the quotient map and $\chi$ is a character of the finite abelian group $\mathbb{Z}_{h}^{s} / G_{h}^{J}$. Thus we established a one-to-one correspondence between the characters of this last group and the elements of $\tilde{\Theta}_{h}(J)$ and our claim follows from the fact that

$$
\left|\mathbb{Z}_{h}^{s} / G_{h}^{J}\right|=\left|\operatorname{det} X_{J}\right|
$$

In the proof of Theorem 1.1 we will also use the following combinatorial result which is due to Dahmen and Micchelli.

Proposition $5.1\left[\mathbf{D M}_{2}\right.$, ThEOREM 3.1]. For every $h>0$ and every $y \in$ $A_{h}(\Gamma)$

$$
\left|b_{h}^{\Gamma}(\underline{y})\right|=\sum_{J \in \mathbf{J}(\Gamma)}\left|\operatorname{det} X_{J}\right| .
$$

Proof of Theorem 1.1. Part (a) was established in Corollary 4.1. Application of Theorem 2.1 to each of the components in (4.11) yields

$$
\operatorname{dim} \mathscr{S}_{h}(\Gamma) \geq \sum_{\underline{\theta} \in \tilde{\Theta}_{h}(\Gamma)}\left|\mathbf{J}\left(\tilde{\Gamma}_{\underline{\theta}, h}\right)\right|,
$$

with equality if and only if

$$
\operatorname{dim} \mathscr{H}\left(\tilde{\Gamma}_{\underline{\theta}, h}\right)=\left|\mathbf{J}\left(\tilde{\Gamma}_{\underline{\theta}, h}\right)\right|, \quad \forall \underline{\theta} \in \tilde{\Theta}_{h}(\Gamma) .
$$

By Lemma 5.1 we know that each $J \in \mathbf{J}(\Gamma)$ lies exactly in $\left|\operatorname{det} X_{J}\right|$ sets from $\left\{\tilde{\Gamma}_{\underline{\theta}, h} \mid \underline{\theta} \in \tilde{\Theta}_{h}(\Gamma)\right\}$, so

$$
\sum_{\underline{\theta} \in \tilde{\Theta}_{h}(\Gamma)}\left|\mathbf{J}\left(\tilde{\Gamma}_{\underline{\theta}, h}\right)\right|=\sum_{J \in \mathbf{J}(\Gamma)}\left|\operatorname{det} X_{J}\right| .
$$


Hence (5.1) implies

$$
\operatorname{dim} \mathscr{S}_{h}(\Gamma) \geq \sum_{J \in \mathbf{J}(\Gamma)}\left|\operatorname{det} X_{J}\right|
$$

whereas Theorem 4.1 says that

$$
\operatorname{dim} \mathscr{S}_{h}(\Gamma) \leq\left|b_{h}^{\Gamma}(\underline{y})\right|, \quad \forall \underline{y} \in A_{h}(\Gamma) .
$$

Therefore, application of Proposition 5.1 completes the proof of (c), which in view of Theorem 4.1 also gives (d).

To prove (b) note that we have just shown that equality holds in (5.1); hence (5.2) is valid for every $h>0$. Thus by appealing to Proposition 4.1 we obtain

$$
\operatorname{dim} \mathscr{H}\left(\Gamma_{\underline{\theta}}\right)=\left|\mathbf{J}\left(\Gamma_{\underline{\theta}}\right)\right|, \quad \forall \underline{\theta} \in \Theta(\Gamma),
$$

and since every $J \in \mathbf{J}(\Gamma)$ is contained exactly in one set from $\left\{\Gamma_{\theta} \mid \underline{\theta} \in \Theta(\Gamma)\right\}$, (b) follows from Theorem 4.2.

Combining Theorem 1.1(b) and Proposition 2.1 one obtains the following result which could also be verified directly:

COROllary 5.1. Assume $\Gamma$ is simple. Then

$$
\mathscr{H}(\Gamma)=\bigoplus_{\underline{\theta} \in \Theta(\Gamma)} e_{\underline{\theta}}(\cdot) .
$$

As a by-product we also derive

THEOREM 5.1. Let $\Gamma$ be a defining set and fix $h>0$. Then the following conditions are equivalent:

(a) To every $u \in \mathscr{S}_{h}(\Gamma)$ there corresponds a unique $f \in \mathscr{H}(\Gamma)$ which interpolates $u(\cdot)\left(\right.$ on $\left.\mathbb{Z}_{h}^{s}\right)$.

$$
\begin{gathered}
\left|\operatorname{det} X_{J}\right|=1, \quad \forall J \in \mathbf{J}(\Gamma), \\
\underline{\theta}^{k}-\underline{\theta}^{j} \notin h^{-1} 2 \pi i \mathbb{Z}^{s} \backslash\{\underline{0}\}, \quad \forall \underline{\theta}^{k}, \underline{\theta}^{j} \in \Theta(\Gamma) .
\end{gathered}
$$

PROOF. Comparing parts (b) and (c) of Theorem 1.1 the necessity of (5.4) is clear. Assuming (5.4) we know from Theorem 1.1 that $\operatorname{dim} \mathscr{S}_{h}(\Gamma)=\operatorname{dim} \mathscr{H}(\Gamma)$ whereas by $\left.(1.11) \mathscr{H}(\Gamma)\right|_{\mathbf{z}_{h}^{s}} \subset \mathscr{S}_{h}(\Gamma)$. So to establish (a) it is necessary and sufficient that restriction to $\mathbb{Z}_{h}^{s}$ will be one to one on $\mathscr{H}(\Gamma)$, which by Theorem 4.2 and Proposition 3.1 is equivalent to (5.5).

Part (d) of Theorem 1.1 can be restated as follows:

COROLlaRY 5.2. For every $\underline{\alpha} \in \mathbb{R}^{s}$ denote by $\delta_{\underline{\alpha}}$ the linear functional of evaluation at $\underline{\alpha}$. Then for every $\underline{x} \in A_{h}(\Gamma)$ the set $\left\{\delta_{\underline{\alpha}}\right\}_{\underline{\alpha} \in b_{h}^{\Gamma}(\underline{x})}$ forms a basis to the dual of $\mathscr{S}_{h}(\Gamma)$. Namely every linear functional $\rho$ on $\mathscr{S}_{h}(\Gamma)$ has a unique representation of the form

$$
\rho(u)=\sum_{\underline{\alpha} \in b_{h}^{\Gamma}(\underline{x})} c_{\underline{\alpha}} u(\underline{\alpha}), \quad \forall u \in \mathscr{S}_{h}(\Gamma) .
$$

Now, when (5.5) holds we know from Proposition 3.1 that $\mathscr{H}(\Gamma)$ is embedded in $\mathscr{S}_{h}(\Gamma)$ and thus Corollary 5.2 yields the following. 
COROLlaRY 5.3. Let $\underline{x} \in A_{h}(\Gamma)$ and assume (5.5) holds. Then every linear functional $\rho$ on $\mathscr{H}(\Gamma)$ has a representation as in (5.6). This representation is unique if and only if (5.4) holds.

Finally we are interested in the case of " $h$-discrete simplicity", namely the case where $\mathscr{S}_{h}(\Gamma)$ is spanned by pure exponentials. In general such requirement is stronger than the usual simplicity. Yet we have

THEOREM 5.2. To every simple defining set $\Gamma$ there corresponds a positive number $h_{0}$, which depends only on $X_{\Gamma}$ and $\left\|\underline{\lambda}_{\Gamma}\right\|_{\infty}$, such that for $h<h_{0}$

$$
\mathscr{S}_{h}(\Gamma)=\bigoplus_{\underline{\theta} \in \tilde{\Theta}_{h}(\Gamma)} e_{\underline{\theta}}(\cdot)
$$

Moreover, if $\underline{\lambda}_{\Gamma} \subset \mathbb{R}$ then (5.7) holds for every $h>0$.

PROOF. If $\Gamma$ does not satisfy (5.7) then there exists $\underline{\theta} \in \tilde{\Theta}_{h}(\Gamma)$ such that $\left|\tilde{\Gamma}_{\underline{\theta}, h}\right| \geq s+1$ and hence contains at least two bases $J_{1}, J_{2}$ from $\mathbf{J}(\Gamma)$. Let $\underline{\theta}^{j}$, $j=1,2$, be the only node of $\Theta\left(J_{j}\right), j=1,2$. By the proof of Lemma 5.1 we know that

$$
\underline{\theta}=\underline{\theta}^{1}+i \underline{\nu}^{1}=\underline{\theta}^{2}+i \underline{\nu}^{2}
$$

where $\underline{\nu}^{1}, \underline{\nu}^{2} \in \mathbb{R}^{s}$ and

$$
e^{h i \underline{\nu}^{j} \cdot \underline{x}_{\gamma}}=1, \quad \forall \gamma \in J_{j}, j=1,2 .
$$

In particular we have $h m\left(\underline{\nu}^{1}-\underline{\nu}^{2}\right) \in 2 \pi \mathbb{Z}^{s}$ where $m$ is the common multiple of $\left\{\left|\operatorname{det} X_{J}\right| \mid J \in \mathbf{J}(\Gamma)\right\}$.

Now, if $\underline{\lambda}_{\Gamma} \subset \mathbb{R}$ then also $\Theta(\Gamma) \subset \mathbb{R}^{s}$, so the equality $\underline{\theta}^{1}+i \underline{\nu}^{1}=\underline{\theta}^{2}+i \underline{\nu}^{2}$ implies $\underline{\theta}^{1}=\underline{\theta}^{2}$ which is impossible since $\Gamma$ is simple. Consequently (5.7) holds in this case for every $h>0$.

For general $\underline{\lambda}_{\Gamma}$ we use the fact that there exists $c>0$ independent of $\underline{\lambda}_{\Gamma}$ such that $\|\underline{\theta}\|_{\infty} \leq c\left\|\underline{\lambda}_{\Gamma}\right\|_{\infty}$ for all $\underline{\theta} \in \Theta(\Gamma)$. Choosing $t_{0}>\left\|\underline{\lambda}_{\Gamma}\right\|_{\infty}$ we take $h_{0}=1 / 2 c m t_{0}$. Then for $h<h_{0}$

$$
\left\|h m i\left(\underline{\nu}^{1}-\underline{\nu}^{2}\right)\right\|_{\infty}=h m\left\|\underline{\theta}^{1}-\underline{\theta}^{2}\right\|_{\infty}<\frac{1}{2 c m t_{0}} 2 m c t_{0}=1,
$$

forcing $\underline{\nu}^{1}=\underline{\nu}^{2}$ and therefore $\underline{\theta}^{1}=\underline{\theta}^{2}$ which again contradicts the simplicity of $\Gamma$. Hence every $\tilde{\tilde{\Gamma}}_{\underline{\theta}, h}, \underline{\theta} \in \tilde{\Theta}_{h}(\Gamma)$, contains exactly $s$ elements and (5.7) follows.

6. Exponential box splines and their related spaces. In this section we apply some of the results of the previous sections to exponential box splines.

Let $\Gamma$ and $h>0$ be given. For a sequence $u(\cdot)$ defined on $\mathbb{Z}_{h}^{s}$ we denote

$$
B_{h}(\Gamma) *_{h} u:=\sum_{\underline{\alpha} \in \mathbb{Z}_{h}^{s}} u(\underline{\alpha}) B_{h}(\Gamma \mid \cdot-\underline{\alpha}) .
$$

The range of the map $B_{h}(\Gamma) *_{h}$ is denoted by $\mathbf{S}_{h}(\Gamma)$ while $\mathbf{H}_{h}(\Gamma)$ stands for the subspace of all entire functions in $\mathbf{S}_{h}(\Gamma)$. Our first claim is the following. 
Proposition 6.1. For any defining set $\Gamma$

$$
\mathbf{H}_{h}(\Gamma) \subset \mathscr{H}(\Gamma) .
$$

ProOF. Assume that $f \in \mathbf{H}_{h}(\Gamma)$. Then $f \in B_{h}(\Gamma) *_{h} u$ for some sequence $u(\cdot)$ defined on $\mathbb{Z}_{h}^{s}$. Let $K \in \mathscr{K}(\Gamma)$. Then by (1.15)

$$
D^{K} f=D^{K}\left(B_{h}(\Gamma) *_{h} u\right)=\left(D^{K} B_{h}(\Gamma)\right) *_{h} u=\nabla_{h}^{K}\left[B_{h}(\Gamma \backslash K) *_{h} u\right] .
$$

Now, $K \in \mathscr{K}(\Gamma)$ means $\langle\Gamma \backslash K\rangle \neq \mathbb{R}^{s}$. Thus (1.4) shows that supp $B_{h}(\Gamma \backslash K)$ lies on a hyperplane of $\mathbb{R}^{s}$. We conclude that $D^{K} f$ is an entire function whose support is contained in a countable union of hyperplanes. Consequently $D^{K} f \equiv 0$, which implies

$$
f \in \bigcap_{K \in \mathscr{K}(\Gamma)} \operatorname{ker} D^{K}=\mathscr{H}(\Gamma)
$$

In certain cases $\mathbf{H}_{h}(\Gamma)$ is a proper subspace of $\mathscr{H}(\Gamma)$ (cf. [ $\mathbf{R}_{2}$, Ex. 4.1]). Conditions for equality in (6.2) will be derived later.

The significance of $\mathscr{S}_{h}(\Gamma)$ in the present analysis is due to the following theorem:

THEOREM 6.1. The map $B_{h}(\Gamma) *_{h}$ carries $\mathscr{S}_{h}(\Gamma)$ onto $\mathbf{H}_{h}(\Gamma)$.

Proof. We first show that $B_{h}(\Gamma) *_{h}$ maps $\mathscr{S}_{h}(\Gamma)$ into $\mathbf{H}_{h}(\Gamma)$. Let $u(\cdot) \in \mathscr{S}_{h}(\Gamma)$ and denote $f_{u}=B_{h}(\Gamma) *_{h} u$. For each $K \in \mathscr{K}(\Gamma)$ we have by (1.15)

$$
D^{K} f_{u}=\left[D^{K} B_{h}(\Gamma)\right] *_{h} u=\left[\nabla_{h}^{K} B_{h}(\Gamma \backslash K)\right] *_{h} u=B_{h}(\Gamma \backslash K) *_{h} \nabla_{h}^{K} u=0 .
$$

Hence $f_{u} \in \mathscr{H}(\Gamma)$. By Theorem 4.2 $\mathscr{H}(\Gamma)$ contains only entire functions. Thus by definition $f_{u} \in \mathbf{H}_{h}(\Gamma)$.

We now prove that this map is onto. Let $f=B_{h}(\Gamma) *_{h} u_{1} \in \mathbf{H}_{h}(\Gamma)$ be arbitrary, where $u_{1}(\cdot): \mathbb{Z}_{h}^{s}$. Choose any $\underline{y} \in A_{h}(\Gamma)$. By Theorem 1.1(d) there exists an element $u_{2} \in \mathscr{S}_{h}(\Gamma)$ satisfying

$$
\left.u_{2}\right|_{b_{h}^{\Gamma}(\underline{y})}=\left.u_{1}\right|_{b_{h}^{\Gamma}(\underline{y})} .
$$

Denote $\tilde{f}=B_{h}(\Gamma) *_{h}\left(u_{2}-u_{1}\right)$. From the first part of the proof $B_{h}(\Gamma) *_{h} u_{2}$ is entire and therefore $\tilde{f}$ is entire. Let $A$ be the $\Gamma-h$ cell containing $\underline{y}$. Then for every $\underline{x} \in A$,

$$
\tilde{f}(\underline{x})=\sum_{\underline{\alpha} \in \mathbb{Z}_{h}^{s}}\left(u_{1}-u_{2}\right)(\underline{\alpha}) B_{h}(\Gamma \mid \underline{x}-\underline{\alpha})=\sum_{\underline{\alpha} \in b_{h}^{\Gamma}(\underline{y})}\left(u_{1}-u_{2}\right)(\underline{\alpha}) B_{h}(\Gamma \mid \underline{x}-\underline{\alpha}),
$$

because $B_{h}(\Gamma \mid \underline{x}-\underline{\alpha})=0$ whenever $\underline{x} \in A$ and $\underline{\alpha} \notin b_{h}^{\Gamma}(\underline{y})$. Taking into account (6.4) we obtain $\left.\tilde{f}\right|_{A} \equiv 0$. Since $\tilde{f}$ is entire the last equality readily implies $\tilde{f} \equiv 0$ on $\mathbb{R}^{s}$. Thus $B_{h}(\Gamma) *_{h} u_{2}=f$.

PROOF OF THEOREM 1.4. The theorem follows from Proposition 6.1 and Theorem 6.1 above.

In the next two results we shall make use of the following lemma:

LEMMA 6.1. Given $\underline{\theta} \in \Theta(\Gamma)$, consider the following two conditions:

(a) There exists $\underline{\theta}^{0} \neq \underline{\theta}$ in $\Theta(\Gamma)$ such that $h\left(\underline{\theta}^{0}-\underline{\theta}\right) \in 2 \pi i \mathbb{Z}^{s}$.

(b) $\hat{B}_{h}(\Gamma \mid-i \underline{\theta})=0$.

Then (a) always implies (b). If in addition we assume

$$
\left|\operatorname{det} X_{J}\right|=1, \quad \forall J \in \mathbf{J}(\Gamma) \text {, }
$$

then (b) implies (a) as well. 
Proof. Assume (a). Since $\underline{\theta}^{0}-\underline{\theta} \neq 0$ and $\left\langle\Gamma_{\underline{\theta}^{0}}\right\rangle=\mathbb{R}^{s}$, there exists $\gamma \in \Gamma_{\underline{\theta}^{0}}$ such that $\underline{x}_{\gamma} \cdot\left(\underline{\theta}-\underline{\theta}^{0}\right) \neq 0$. But $\underline{x}_{\gamma} \in \mathbb{Z}^{s}$. Therefore $h\left(\lambda_{\gamma}-\underline{x}_{\gamma} \cdot \underline{\theta}\right)=h \underline{x}_{\gamma} \cdot\left(\underline{\theta}^{0}-\underline{\theta}\right) \in$ $2 \pi i \mathbb{Z} \backslash\{0\}$. This implies that $\int_{0}^{h} e^{\left(\lambda_{\gamma}-\underline{x}_{\gamma} \cdot \underline{\theta}\right) t} d t=0$, which in view of (1.2) gives $\hat{B}_{h}(\Gamma \mid-i \underline{\theta})=0$. that

For the converse assume $\hat{B}_{h}(\Gamma \mid-i \underline{\theta})=0$. Then by (1.2) there exists $\gamma_{0} \in \Gamma$ such

$$
h\left(\lambda_{\gamma_{0}}-\underline{x}_{\gamma_{0}} \cdot \underline{\theta}\right) \in 2 \pi i \mathbb{Z} \backslash\{0\} .
$$

Complete $\gamma_{0}$ to a basis $J_{0} \in \mathbf{J}(\Gamma)$ by elements of $\Gamma_{\underline{\theta}}$ and let $\underline{\theta}^{0}$ be the node in $\Theta(\Gamma)$ which satisfies $\underline{x}_{\gamma} \cdot \underline{\theta}^{0}-\lambda_{\gamma}=0$ for every $\gamma \in J_{0}$. Given $\gamma \in J_{0} \backslash \gamma_{0}$ we know that $\gamma \in \Gamma_{\underline{\theta}} \cap \Gamma_{\underline{\theta}^{0}}$ and therefore

$$
h\left(\underline{x}_{\gamma} \cdot\left(\underline{\theta}^{0}-\underline{\theta}\right)\right)=h\left(\lambda_{\gamma}-\lambda_{\gamma}\right)=0 .
$$

From (6.6) and the last equation we conclude that $h\left(\underline{\theta}^{0}-\underline{\theta}\right) / 2 \pi i$ coincides with an integer multiple of one of the columns of $\left(X_{J_{0}}\right)^{-1}$, which, since $\left|\operatorname{det} X_{J_{0}}\right|=1$, contains only integer entries. This proves (a).

Given $f \in \mathscr{H}(\Gamma)$ we know from (1.11) that $\left.f\right|_{\mathbf{Z}_{h}^{s}} \in \mathscr{S}_{h}(\Gamma)$. Hence Theorem 6.1 yields

$$
B_{h}(\Gamma) *_{h}\left(\left.f\right|_{\mathbf{z}_{h}^{s}}\right) \in \mathbf{H}_{h}(\Gamma), \quad \forall f \in \mathscr{H}(\Gamma) .
$$

The next result gives a necessary and sufficient condition for $B_{h}(\Gamma) *_{h}$ being injective on $\mathscr{H}(\Gamma) \mid \mathbf{z}_{h}^{s}$.

THEOREM 6.2. Define a map $B_{h}(\Gamma) *_{h}^{\prime}: C\left(\mathbb{R}^{s}\right)$ by

$$
B_{h}(\Gamma) *_{h}^{\prime}: f \rightarrow B_{h}(\Gamma) *_{h}\left(\left.f\right|_{\mathbf{Z}_{h}^{s}}\right) .
$$

Let $\Gamma_{1} \subset \Gamma$ and assume $\left\langle\Gamma_{1}\right\rangle=\mathbb{R}^{s}$. Then

(a) $\mathscr{H}\left(\Gamma_{1}\right)$ is an invariant subspace of $B_{h}(\Gamma) *_{h}^{\prime}$.

(b) $B_{h}(\Gamma) *_{h}^{\prime}$ is an automorphism of $\mathscr{H}\left(\Gamma_{1}\right)$ if and only if

$$
\hat{B}_{h}(\Gamma \mid-i \underline{\theta}) \neq 0, \quad \forall \underline{\theta} \in \Theta\left(\Gamma_{1}\right) .
$$

Proof. Let $f \in \mathscr{H}\left(\Gamma_{1}\right)$ and let $K \in \mathscr{K}\left(\Gamma_{1}\right)$. Then $D^{K} f \equiv 0$ and therefore by (1.11) $\nabla_{h}^{K} f \equiv 0$. Hence (as in the proof of the first part of Theorem 6.1)

$$
D^{K}\left(B_{h}(\Gamma) *_{h}^{\prime} f\right)=B_{h}(\Gamma \backslash K) *_{h}^{\prime} \nabla_{h}^{K} f \equiv 0 .
$$

This proves (a).

Now denote

$$
\operatorname{Ker}_{h}\left(\Gamma_{1}\right)=\left\{f \in \mathscr{H}\left(\Gamma_{1}\right) \mid B_{h}(\Gamma) *_{h}^{\prime} f=0\right\},
$$

and assume (6.8) holds. By Lemma 6.1 we know that

$$
h\left(\underline{\theta}^{1}-\underline{\theta}^{2}\right) \notin 2 \pi i \mathbb{Z}^{s} \backslash\{\underline{0}\}, \quad \forall \underline{\theta}^{1}, \underline{\theta}^{2} \in \Theta\left(\Gamma_{1}\right) .
$$

Hence we can combine Theorem 4.2, Proposition 3.1, Corollary 3.1 and the obvious fact that $\left.\operatorname{Ker}_{h}\left(\Gamma_{1}\right)\right|_{\mathbf{z}_{h}^{s}}$ is shift-invariant to conclude that if $\operatorname{Ker}_{h}\left(\Gamma_{1}\right)$ is not trivial 
then it must contain an exponential $e_{\underline{\theta}^{0}}, \underline{\theta}^{0} \in \Theta\left(\Gamma_{1}\right)$. Now we appeal to $\left[\mathbf{R}_{1}\right.$, Corollary 5.1] which states that

$$
B_{h}(\Gamma) *_{h}^{\prime} e_{\underline{\theta}}=h^{-s} \hat{B}_{h}(\Gamma \mid-i \underline{\theta}) e_{\underline{\theta}}, \quad \forall \underline{\theta} \in \Theta(\Gamma),
$$

to conclude that $\hat{B}_{h}\left(\Gamma \mid-i \underline{\theta}^{0}\right)=0$, which contradicts $(6.8)$. Consequently $B_{h}(\Gamma) *_{h}^{\prime}$ is injective on $\mathscr{H}\left(\Gamma_{1}\right)$ and therefore by the first part of the proof induces an automorphism on $\mathscr{H}\left(\Gamma_{1}\right)$.

For the converse assume (6.8) is false. Then $\hat{B}_{h}(\Gamma \mid-i \underline{\theta})=0$ for some $\underline{\theta} \in \Theta\left(\Gamma_{1}\right)$. Taking (6.9) into account we see that $e_{\theta} \in \operatorname{Ker}_{h}(\Gamma)$. By Theorem $4.2 e_{\underline{\theta}} \in \mathscr{H}\left(\Gamma_{1}\right)$ and hence $B_{h}(\Gamma) *_{h}^{\prime}$ is not injective on $\overline{\mathscr{H}}\left(\Gamma_{1}\right)$.

COROllary 6.1. Assume that

$$
\hat{B}_{h}(\Gamma \mid-i \underline{\theta}) \neq 0, \quad \forall \underline{\theta} \in \Theta(\Gamma) .
$$

Then

$$
\mathbf{H}_{h}(\Gamma)=\mathscr{H}(\Gamma) .
$$

Application of Theorem 1.1(b) gives

COROllary 6.2. Assume (6.10) holds. Then $\operatorname{dim} \mathbf{H}_{h}(\Gamma)=|\mathbf{J}(\Gamma)|$.

For polynomial box splines (namely in the case when $\left.\underline{\lambda}_{\Gamma}=\underline{0}\right) \Theta(\Gamma)=\{\underline{0}\}$ and $\hat{B}_{h}(\Gamma \mid \underline{0})=h^{|\Gamma|} \neq 0$. Therefore in this case (6.11) always holds. This result is due to de Boor and Höllig, $[\mathbf{B H}]$, and proved by a different method in $\left[\mathbf{D M}_{\mathbf{1}}\right]$. The fact that (6.11) is valid for polynomial box splines was used in $\left[\mathbf{R}_{2}\right]$ to establish a similar result to that of Corollary 6.1. We also mention that (6.10) is not a necessary condition for (6.11) (cf. [ $\mathbf{R}_{2}$, Ex. 4.2]).

Although Corollary 6.1 is valid for every choice of $\Gamma$, a relatively simpler proof exists for the choice $\underline{\lambda}_{\Gamma} \subset \mathbb{R}$. By Corollary 4.2 we know that (6.10) is always valid in this case. Hence

COROllary 6.1*. Assume $\underline{\lambda}_{\Gamma} \subset \mathbb{R}$. Then $\mathbf{H}_{h}(\Gamma)=\mathscr{H}(\Gamma)$.

Proof. By Theorem 6.2(a) we know that for each $\underline{\theta} \in \Theta(\Gamma), B_{h}(\Gamma) *_{h}^{\prime}$ maps $\mathscr{H}\left(\Gamma_{\underline{\theta}}\right)$ into itself. Since $\underline{\lambda}_{\Gamma} \subset \mathbb{R}$ it follows that $B_{h}(\Gamma)$ is positive and therefore (as mentioned in [BD]) $B_{h}(\Gamma) *_{h}^{\prime}$ is injective on polynomials. But by Theorem 4.2 $\mathscr{H}\left(\Gamma_{\underline{\theta}}\right)=e_{\underline{\theta}} \mathscr{H}\left(X_{\underline{\theta}}\right)$ where $\mathscr{H}\left(X_{\underline{\theta}}\right)$ is a finite dimensional polynomial space. Now the desired result readily follows.

Finally we derive necessary and sufficient conditions for $\mathscr{S}_{h}(\Gamma)$ being isomorphic to $\mathbf{H}_{h}(\Gamma)$

THEOREM 6.3. The following conditions are equivalent:

(a) $B_{h}(\Gamma) *_{h}$ induces isomorphism between $\mathscr{S}_{h}(\Gamma)$ and $\mathbf{H}_{h}(\Gamma)$.

(b) $\mathbf{H}_{h}(\Gamma)=\mathscr{H}(\Gamma)$ and $\left|\operatorname{det} X_{J}\right|=1$ for all $J \in \mathbf{J}(\Gamma)$.

(c) $\hat{B}_{h}(\Gamma \mid-i \underline{\theta}) \neq 0$ for all $\underline{\theta} \in \Theta(\Gamma)$ and $\left|\operatorname{det} X_{J}\right|=1$ for all $J \in \mathbf{J}(\Gamma)$.

(d) $h\left(\underline{\theta}^{1}-\underline{\theta}^{2}\right) \notin 2 \pi i \mathbb{Z}^{s} \backslash\{\underline{0}\}$ for all $\underline{\theta}^{1}, \underline{\theta}^{2} \in \Theta(\Gamma)$ and $\left|\operatorname{det} X_{J}\right|=1$ for all $J \in \mathbf{J}(\Gamma)$.

ProOF. We prove the theorem by showing (d) $\Leftrightarrow(\mathrm{c}) \Rightarrow(\mathrm{b}) \Rightarrow(\mathrm{a}) \Rightarrow(\mathrm{c})$. The equivalence $(\mathrm{c}) \Leftrightarrow(\mathrm{d})$ follows from the Lemma 6.1. Corollary 6.1 shows that (c) 
.mplies (b). Assuming (b) we conclude from Theorem 1.1(b), (c) that $\operatorname{dim} \mathscr{S}_{h}(\Gamma)=$ $\operatorname{dim} \mathscr{H}(\Gamma)=\operatorname{dim} \mathbf{H}_{h}(\Gamma)$, and (a) follows in this case from Theorem 6.1. Finally assume that (a) holds. Then, $\operatorname{dim} \mathscr{S}_{h}(\Gamma)=\operatorname{dim} \mathbf{H}_{h}(\Gamma)$ which in view of Theorem 1.1(b), (c) and Proposition 6.1 implies $\operatorname{dim} \mathscr{S}_{h}(\Gamma)=\operatorname{dim} \mathscr{H}(\Gamma)$. Referring again to Theorem 1.1(b), (c) we conclude $\left|\operatorname{det} X_{J}\right|=1$ for all $J \in \mathbf{J}(\Gamma)$.

To show that (c) holds, let $\underline{\theta} \in \Theta(\Gamma)$. By (1.11) and Proposition 2.1, $e_{\underline{\theta}} \mid \mathbf{z}_{h}^{s} \in$ $\mathscr{S}_{h}(\Gamma)$. Since we assume (a) $B_{h}(\Gamma) *_{h}\left(e_{\underline{\theta}} \mid \mathbf{z}_{h}^{s}\right) \neq 0$, therefore (6.9) gives $\hat{B}_{h}(\Gamma \mid-i \underline{\theta}) \neq$ 0 . Consequently (c) is valid and the proof of the theorem is completed.

7. Analysis of $H(\Gamma)$. To simplify the notations we assume throughout this section that $h=1$ and omit this subscript. Yet, all the results here are valid for arbitrary $h$ with the obvious modifications.

Our main aim is to derive necessary and sufficient conditions for the equality

$$
\mathbf{H}(\Gamma)=\mathscr{H}(\Gamma) .
$$

Utilizing these conditions we also obtain a formula for $\operatorname{dim} \mathbf{H}(\Gamma)$.

In order to analyze $\mathbf{H}(\Gamma)$, it suffices, by Theorem 6.1 , to examine the range of $\mathscr{S}(\Gamma)$ under $B(\Gamma) *$. To study the action of $B(\Gamma) *$ on $\mathscr{S}(\Gamma)$ we make use of the direct sum decomposition of Theorem 4.3 and investigate the action of $B(\Gamma) *$ on each summand. For this purpose denote for each $\underline{\tilde{\theta}} \in \tilde{\Theta}(\Gamma)$

$$
\begin{gathered}
\mathscr{S}_{\underline{\hat{\theta}}}:=e_{\underline{\tilde{\theta}}} \mathscr{H}\left(\tilde{X}_{\tilde{\theta}}\right) \mid \mathbf{z}^{s}=\left\{e_{\underline{\tilde{\theta}}}(\underline{\alpha}) p(\underline{\alpha}) \mid p \in \mathscr{H}\left(\tilde{X}_{\tilde{\theta}}\right)\right\}, \\
\mathbf{H}_{\underline{\tilde{\theta}}}=\left\{B(\Gamma) * u \mid u \in \mathscr{S}_{\underline{\hat{\theta}}}\right\}, \\
{[\underline{\tilde{\theta}}]=\left\{\underline{\theta} \in \Theta(\Gamma) \mid \underline{\tilde{\theta}}-\underline{\theta} \in 2 \pi i \mathbb{Z}^{s}\right\} .}
\end{gathered}
$$

Using these notations, Theorem 4.3 can be written as

$$
\mathscr{S}(\Gamma)=\bigoplus_{\tilde{\tilde{\theta}} \in \tilde{\Theta}(\Gamma)} \mathscr{S}_{\underline{\underline{\theta}}}
$$

while Theorem 6.1 implies

$$
\mathbf{H}(\Gamma)=\left\langle\bigcup_{\underline{\tilde{\theta}} \in \tilde{\Theta}(\Gamma)} \mathbf{H}_{\underline{\tilde{\theta}}}\right\rangle .
$$

Our first result gives significant information on $\mathbf{H}_{\underline{\tilde{\theta}}}$ in terms of $[\underline{\tilde{\theta}}]$.

Proposition 7.1. For every $\underline{\tilde{\theta}} \in \tilde{\Theta}(\Gamma)$

$$
\mathbf{H}_{\underline{\tilde{\theta}}} \subset \bigoplus_{\underline{\theta} \in[\underline{\tilde{\theta}}]} \mathscr{H}\left(\Gamma_{\underline{\theta}}\right) .
$$

PrOOF. Let $u(\underline{\alpha})=e_{\underline{\tilde{\theta}}}(\underline{\alpha}) p(\underline{\alpha}) \in \mathscr{S}_{\underline{\hat{\theta}}}$. By Proposition $6.1 \mathbf{H}(\Gamma) \subset \mathscr{H}(\Gamma)$ and therefore Theorem 4.2 implies

$$
B(\Gamma) * u=\sum_{\underline{\theta}^{j} \in \Theta(\Gamma)} e_{\underline{\theta}^{j}}(\underline{x}) p_{j}(\underline{x}), \quad p_{j} \in \pi .
$$


Now, if $\underline{\theta}^{j_{0}} \notin[\underline{\tilde{\theta}}]$ then, as in the proof of Lemma 3.1(b), one can find a difference operator $\Delta$ (on $\mathbb{Z}^{s}$ ) which on the one hand annihilates $u(\cdot)$ and on the other hand is injective on $\mathscr{H}\left(\Gamma_{\underline{\theta}^{j}}\right)$. Application of $\Delta$ to both sides of (7.8) gives

$$
\sum_{\underline{\theta}^{j} \in \Theta(\Gamma)} \Delta\left(e_{\underline{\theta}^{j}}(\underline{x}) p_{j}(\underline{x})\right)=B(\Gamma) * \Delta u=0 .
$$

Since $\Delta$ is injective on $\mathscr{H}\left(\Gamma_{\theta^{j_{0}}}\right)$ one concludes $p_{j_{0}}=0$.

REMARK 7.1. Note that the only information used in the proof of Proposition 7.1 was that $u(\cdot)$ is an exponential polynomial, while $B(\Gamma) * u$ is a linear combination of exponential polynomials. Thus, replacing $B(\Gamma)$ by an arbitrary compactly supported $\phi \in \mathscr{D}^{\prime}\left(\mathbb{R}^{s}\right)$ and assuming that $B(\Gamma) * u$ is a linear combination of $P E$ functions one can easily establish a generalization of Proposition 7.1 with respect to $\phi$.

An immediate consequence of Proposition 7.1 is the following

Corollary 7.1. Assume $\tilde{\tilde{\theta}} \in \tilde{\Theta}(\Gamma)$ and $[\underline{\tilde{\theta}}]=\varnothing$. Then $\mathbf{H}_{\underline{\tilde{\theta}}}=0$.

REMARK 7.2. Corollary 7.1 covers Theorem 4.2 of $\left[\mathbf{D M}_{3}\right]$ and Theorem 7.1 of $\left[\mathbf{R}_{1}\right]$ as special cases.

REMARK 7.3. In case (6.10) holds we know from (4.13) and Theorem 4.3 that

$$
\mathscr{S}(\Gamma)=\mathscr{H}(\Gamma) \oplus E(\Gamma),
$$

where $E(\Gamma)=\bigoplus_{\underline{\tilde{\theta}} \in \tilde{\Theta}(\Gamma) ;[\tilde{\tilde{\theta}}]=\varnothing} \mathscr{S}_{\underline{\tilde{\theta}}}$. Thus, by Theorem 6.2 and Corollary 7.1 we see that (7.9) expresses $\mathscr{S}(\bar{\Gamma})$ as a direct sum of two subspaces for which $B(\Gamma) *$ is injective on the first and annihilates the other.

Since each $\underline{\theta} \in \Theta(\Gamma)$ lies exactly in a unique equivalence class $[\underline{\tilde{\theta}}], \underline{\tilde{\theta}} \in \tilde{\Theta}(\Gamma)$, Proposition 7.1 leads to the conclusion

COROLlary 7.2. $\mathbf{H}(\Gamma)=\bigoplus_{\underline{\tilde{\theta}} \in \tilde{\Theta}(\Gamma)} \mathbf{H}_{\underline{\tilde{\theta}}}$.

The results obtained so far express certain limitations on $\mathbf{H}_{\underline{\tilde{\theta}}}$. The next proposition takes the opposite direction.

Proposition 7.2. Let $\underline{\tilde{\theta}} \in \tilde{\Theta}(\Gamma)$ and let $\underline{\theta} \in[\underline{\tilde{\theta}}]$. Then the operator $D^{\tilde{\Gamma}_{\underline{\underline{\theta}}} \mid \Gamma_{\underline{\theta}}}$ maps $\mathbf{H}_{\underline{\tilde{\theta}}}$ onto $\mathscr{H}\left(\Gamma_{\underline{\theta}}\right)$.

PROOF. In the proof of Proposition 7.2 we make use of the following lemma:

LEMMA 7.1. For each $K_{1} \subset K_{2} \subset \Gamma$, the operator $\nabla^{X_{K_{1}}}$ maps $\mathscr{H}\left(X_{K_{1}}\right)$ onto $\mathscr{H}\left(X_{K_{1} \backslash K_{2}}\right)$.

The proof of Lemma 7.1 is based on

LEMMA 7.2. For each $\gamma \in \Gamma, D^{\gamma}$ maps $\mathscr{H}(\Gamma)$ onto $\mathscr{H}(\Gamma \backslash \gamma)$.

ProOF OF LeMMA 7.2. It is easy to see that $D^{\gamma}$ maps $\mathscr{H}(\Gamma)$ into $\mathscr{H}(\Gamma \backslash \gamma)$. To prove that this map is onto fix $f \in \mathscr{H}(\Gamma \backslash \gamma)$ and let $h>0$ be such that (6.10) holds. (The existence of such $h$ is obvious; It is also guaranteed by Corollary 4.2.) Let $A$ be any $\Gamma-h$ cell and let $\underline{y} \in A$. By Corollary $6.1 \mathbf{H}_{h}(\Gamma \backslash \gamma)=\mathscr{H}(\Gamma \backslash \gamma)$ so there exists $\left\{c_{\underline{\alpha}}\right\}_{\underline{\alpha} \in b_{h}^{\Gamma \backslash \gamma}(\underline{y})}$ such that

$$
\left.f\right|_{A}=\left.\sum_{\underline{\alpha} \in b_{h}^{\Gamma \backslash \gamma}(\underline{y})} c_{\underline{\alpha}} B_{h}(\Gamma \backslash \gamma \mid \cdot-\underline{\alpha})\right|_{A} .
$$


Making use of (1.15) we see that for every $\underline{\alpha} \in \mathbb{Z}_{h}^{s}$

$$
\begin{aligned}
B_{h}(\Gamma \backslash \gamma \mid \cdot-\underline{\alpha}) & =\sum_{j=0}^{\infty} e^{j h \lambda_{\gamma}} \nabla_{h}^{\gamma} B_{h}\left(\Gamma \backslash \gamma \mid \cdot-\underline{\alpha}-j h \underline{x}_{\gamma}\right) \\
& =\sum_{j=0}^{\infty} e^{j h \lambda_{\gamma}} D^{\gamma} B_{h}\left(\Gamma \mid \cdot-\underline{\alpha}-j h \underline{x}_{\gamma}\right) .
\end{aligned}
$$

The last equation when combined with (7.10) shows that

$$
\left.f\right|_{A} \in\left\langle\left\{\left.D^{\gamma} B_{h}(\Gamma \mid \cdot-\underline{\alpha})\right|_{A} \mid \underline{\alpha} \in \mathbb{Z}_{h}^{s}\right\}\right\rangle,
$$

while from [ $\mathbf{R}_{1}$, Corollary 4.3] (or alternately from the proof of Proposition 6.1) we know that $\left.B_{h}(\Gamma \mid \cdot-\underline{\alpha})\right|_{A} \in \mathscr{H}(\Gamma), \forall \underline{\alpha} \in \mathbb{Z}_{h}^{s}$. Hence $\left.f\right|_{A} \in\left\{D^{\gamma} g \mid g \in \mathscr{H}(\Gamma)\right\}$. Since $\mathscr{H}(\Gamma)$ consists of entire functions this last result readily extends to $\mathbb{R}^{s}$ and Lemma 7.2 is established.

PROOF OF LEMMA 7.1. By Theorem $4.2 \mathscr{H}\left(X_{K_{2}}\right)$ contains only polynomials, whereas Lemma 4.1 and (1.11) show that

$$
\nabla^{X_{K_{1}}} p=0 \Leftrightarrow D^{X_{K_{1}}} p=0, \quad \forall p \in \pi .
$$

Therefore $\operatorname{ker}\left(\left.\nabla^{X_{K_{1}}}\right|_{\mathscr{H}\left(X_{K_{2}}\right)}\right)=\operatorname{ker}\left(\left.D^{X_{K_{1}}}\right|_{\mathscr{H}}\left(X_{K_{2}}\right)\right)$ and the claim now follows by repeated use of Lemma 7.2.

To prove Proposition 7.2 denote $\Gamma_{0}=\tilde{\Gamma}_{\underline{\tilde{\theta}}} \backslash \Gamma_{\underline{\theta}}$ and let $u(\cdot)=e_{\underline{\tilde{\theta}}}(\cdot) p(\cdot) \in \mathscr{S}_{\underline{\tilde{\theta}}}$. By

$$
D^{\Gamma_{0}}(B(\Gamma) * u)=B\left(\Gamma \backslash \Gamma_{0}\right) * \nabla^{\Gamma_{0}} u \text {. }
$$

Since $\Gamma_{0} \subset \tilde{\Gamma}_{\tilde{\tilde{\theta}}}$ and $\underline{\theta}-\underline{\tilde{\theta}} \in 2 \pi i \mathbb{Z}^{s}$ then

$$
\nabla^{\Gamma_{0}} u(\underline{\alpha})=e_{\underline{\tilde{\theta}}}(\underline{\alpha}) \nabla^{X_{\Gamma_{0}}} p(\underline{\alpha})=e_{\underline{\theta}}(\underline{\alpha}) \nabla^{X_{\Gamma_{0}}} p(\underline{\alpha}) \text {. }
$$

Also, from the definition of $\Gamma_{0}$ we see that $\hat{B}\left(\Gamma \backslash \Gamma_{0} \mid-i \underline{\theta}\right) \neq 0$ and therefore we can appeal to Theorem 6.2 to conclude that $\hat{B}\left(\Gamma \backslash \Gamma_{0}\right) *^{\prime}$ is an automorphism of $\mathscr{H}\left(\Gamma_{\underline{\theta}}\right)$. Thus (7.11), (7.12) and Lemma 7.1 can be combined to yield

$$
\begin{aligned}
\operatorname{Range}\left(\left.D^{\Gamma_{0}}\right|_{\mathbf{H}_{\underline{\tilde{\theta}}}}\right) & =\left\{D^{\Gamma_{0}}(B(\Gamma) * u) \mid u \in \mathscr{S}_{\underline{\underline{\theta}}}\right\} \\
& =\left\{B\left(\Gamma \backslash \Gamma_{0}\right) * \nabla^{\Gamma_{0}} u \mid u \in \mathscr{S}_{\underline{\hat{\theta}}}\right\} \\
& =\left\{B\left(\Gamma \backslash \Gamma_{0}\right) * u \mid u \in e_{\tilde{\hat{\theta}}} \mathscr{H}\left(X_{\underline{\theta}}\right)\right\} \\
& =\left\{B\left(\Gamma \backslash \Gamma_{0}\right) *^{\prime} f \mid f \in \mathscr{H}\left(\Gamma_{\underline{\theta}}\right)\right\}=\mathscr{H}\left(\Gamma_{\underline{\theta}}\right) .
\end{aligned}
$$

This completes the proof of Proposition 7.2.

Another proof of Lemma 7.1, which does not make use of box splines, is given in [DR].

We now combine together Propositions 7.1 and 7.2 to obtain the following sufficient condition for (7.1).

THEOREM 7.1. Assume that for every distinct $\underline{\theta}^{1}, \underline{\theta}^{2} \in \Theta(\Gamma)$

$$
\underline{\theta}^{1}-\underline{\theta}^{2} \notin 2 \pi i \mathbb{Z}^{s} \text {. }
$$

Then $\mathbf{H}(\Gamma)=\mathscr{H}(\Gamma)$. 
ProOF. By Proposition $6.1 \mathrm{H}(\Gamma) \subset \mathscr{H}(\Gamma)$. To prove the opposite inclusion it is sufficient, in view of Theorem 4.2, to show that for each $\underline{\theta} \in \Theta(\Gamma), \mathscr{H}\left(\Gamma_{\underline{\theta}}\right) \subset \mathbf{H}(\Gamma)$. Fix $\underline{\theta} \in \Theta(\Gamma)$ and let $\underline{\tilde{\theta}} \in \tilde{\Theta}(\Gamma)$ be such that $\underline{\theta} \in[\underline{\tilde{\theta}}]$. By (7.13) $\underline{\theta}$ is the unique node in $[\underline{\tilde{\theta}}]$ and therefore Proposition 7.1 shows that $\mathbf{H}_{\tilde{\theta}} \subset \mathscr{H}\left(\Gamma_{\underline{\theta}}\right)$. But Proposition 7.2 ensures the existence of a linear map from $\mathbf{H}_{\underline{\tilde{\theta}}}$ onto $\mathscr{H}\left(\Gamma_{\underline{\theta}}\right)$. Hence $\mathscr{H}\left(\Gamma_{\underline{\theta}}\right)=\mathbf{H}_{\underline{\tilde{\theta}}} \subset$ $\mathbf{H}(\Gamma)$ and the desired inclusion now follows.

PROOF OF THEOREM 1.5. The theorem follows from Lemma 6.1 and Theorem 7.1 .

REMARK 7.4. Note that in view of Lemma 6.1, Theorem 7.1 is stronger than Corollary 6.1.

For later reference we also record the following result which has been obtained in the proof of Theorem 7.1.

COROllary 7.3. Assume $\underline{\tilde{\theta}} \in \tilde{\Theta}(\Gamma)$ and $[\underline{\tilde{\theta}}]$ consists of one element $\underline{\theta} \in \Theta(\Gamma)$. Then $\mathbf{H}_{\underline{\tilde{\theta}}}=\mathscr{H}\left(\Gamma_{\underline{\theta}}\right)$.

To advance the analysis of $\mathbf{H}(\Gamma)$ we attach to each $\underline{\tilde{\theta}} \in \tilde{\Theta}(\Gamma)$ the following subspace of $\mathscr{S}_{\underline{\hat{\theta}}}$

$$
\mathscr{L}_{\underline{\underline{\theta}}}=\left\{u \in \mathscr{S}_{\underline{\underline{\theta}}} \mid \nabla^{\tilde{\Gamma}_{\overline{\underline{\theta}}} \mid \Gamma_{\underline{\theta}}} u=0 \forall \underline{\theta} \in[\underline{\tilde{\theta}}]\right\} .
$$

By convention if $[\tilde{\theta}]=\varnothing$ we set $\mathscr{L}_{\underline{\underline{\theta}}}=\mathscr{S}_{\underline{\underline{\hat{\theta}}}}$.

The importance of $\mathscr{L}_{\underline{\hat{\theta}}}$ becomes clear as as soon as we know

LEMma 7.3. For $u \in \mathscr{S}_{\underline{\hat{\theta}}}$

$$
B(\Gamma) * u=0 \Leftrightarrow u \in \mathscr{L}_{\underline{\hat{\tilde{\theta}}}} .
$$

Proof. In case $[\underline{\tilde{\theta}}]=\varnothing,(7.15)$ follows from Corollary 7.1 and the definition of $\mathscr{L}_{\underline{\tilde{\theta}}}$. Assume $[\underline{\tilde{\theta}}] \neq \varnothing$. From Proposition 7.1 we know that

$$
B(\Gamma) * u=\sum_{\underline{\theta}^{j} \in[\underline{\tilde{\theta}}]} e_{\underline{\theta}^{j}}(\underline{x}) p_{j}(\underline{x}) .
$$

Fix $\underline{\theta}^{j} \in[\underline{\tilde{\theta}}]$ and denote $\Gamma_{j}=\tilde{\Gamma}_{\underline{\tilde{\theta}}} \backslash \Gamma_{\underline{\theta}^{j}}$. Then by (1.15)

$$
D^{\Gamma_{j}}(B(\Gamma) * u)=B\left(\Gamma \backslash \Gamma_{j}\right) * \nabla^{\Gamma_{j}} u .
$$

Now, if $u \in \mathscr{L}_{\underline{\underline{\theta}}}$ then by definition $\nabla^{\Gamma_{j}} u=0$. Thus (7.16) implies $D^{\Gamma_{j}}(B(\Gamma) * u)=$ 0 . Since $\Gamma_{j} \cap \Gamma_{\underline{\theta}^{j}}=\varnothing$ we know (as in the proof of Theorem 4.2) that $D^{\Gamma_{j}}$ is injective on $\mathscr{H}\left(\bar{\Gamma}_{\underline{\theta}^{j}}\right)$, and consequently $p_{j}=0$. Since $j$ was arbitrary we conclude $B(\Gamma) * u=0$.

For the converse implication, assume $B(\Gamma) * u=0$. Then by (7.16) $B\left(\Gamma \backslash \Gamma_{j}\right) *$ $\nabla^{\Gamma_{j}} u=0$. But $\left.\nabla^{\Gamma_{j}} u \in \mathscr{H}\left(\Gamma_{\underline{\theta}^{j}}\right)\right|_{\mathbb{Z}^{s}}$ while $\hat{B}\left(\Gamma \backslash \Gamma_{j} \mid-i \underline{\theta}^{j}\right) \neq 0$. Hence by appealing to Theorem 6.2 we obtain $\nabla^{\Gamma_{j}} u=0$ and since $j$ was arbitrary we conclude $u \in \mathscr{L}_{\underline{\hat{\theta}}}$.

Our next result is a necessary and sufficient condition for (7.1). 
THEOREM 7.2. $\mathbf{H}(\Gamma)$ is a proper subspace of $\mathscr{H}(\Gamma)$ if and only if there exists $\underline{\tilde{\theta}} \in \tilde{\Theta}(\Gamma)$ which satisfies

$$
\operatorname{dim} \mathscr{L}_{\underline{\underline{\theta}}}>\left|\mathbf{J}\left(\tilde{\Gamma}_{\tilde{\underline{\theta}}}\right)\right|-\sum_{\underline{\theta} \in[\tilde{\tilde{\theta}}]}\left|\mathbf{J}\left(\Gamma_{\underline{\theta}}\right)\right| .
$$

Moreover, for such $\underline{\tilde{\theta}}$ one must have $|[\underline{\tilde{\theta}}]|>1$.

ProOF. By Theorem 6.1

$$
\mathbf{H}(\Gamma)=\operatorname{Range}\left(\left.B(\Gamma) *\right|_{\mathscr{S}}(\Gamma)\right),
$$

while Corollaries $7.1,7.2$ imply that

$$
\mathbf{H}(\Gamma)=\bigoplus_{\tilde{\tilde{\theta}} \in \tilde{\Theta}(\Gamma)} \mathbf{H}_{\tilde{\tilde{\theta}}}=\bigoplus_{\underline{\tilde{\theta}} \in \tilde{\Theta}(\Gamma)} \operatorname{Range}\left(B(\Gamma) * \mid \mathcal{S}_{\underline{\underline{\hat{\theta}}}}\right) .
$$

$$
\mathbf{H}_{\underline{\tilde{\theta}}} \subset \bigoplus_{\underline{\theta} \in[\underline{\tilde{\theta}}]} \mathscr{H}\left(\Gamma_{\underline{\theta}}\right) .
$$

In addition we know that $\mathscr{H}(\Gamma)=\bigoplus_{\underline{\theta} \in \Theta(\Gamma)} \mathscr{H}\left(\Gamma_{\underline{\theta}}\right)$ while each $\underline{\theta} \in \Theta(\Gamma)$ is contained in a unique equivalence class $[\tilde{\theta}]$. Therefore we see that (7.1) fails to hold if and only if for some $\underline{\tilde{\theta}} \in \tilde{\Theta}(\Gamma)$

$$
\operatorname{dim} \mathbf{H}_{\underline{\tilde{\theta}}}<\operatorname{dim}\left(\bigoplus_{\underline{\theta} \in[\underline{\tilde{\theta}}]} \mathscr{H}\left(\Gamma_{\underline{\theta}}\right)\right),
$$

and the definition of $\mathbf{H}_{\underline{\tilde{\theta}}}$ gives

$$
\operatorname{dim} \mathbf{H}_{\underline{\tilde{\theta}}}=\operatorname{dim} \mathscr{S}_{\underline{\tilde{\theta}}}-\operatorname{dim} \operatorname{ker}\left(\left.B(\Gamma) *\right|_{\mathscr{S}_{\hat{\theta}}}\right) .
$$

Now, by Theorem 1.1(b) $\operatorname{dim} \mathscr{S}_{\underline{\underline{\theta}}}=\operatorname{dim} \mathscr{H}\left(\tilde{X}_{\tilde{\underline{\theta}}}\right)=\left|\mathbf{J}\left(\tilde{\Gamma}_{\tilde{\tilde{\theta}}}\right)\right|$ and $\operatorname{dim} \bigoplus_{\underline{\theta} \in[\tilde{\tilde{\theta}}]} \mathscr{H}\left(\Gamma_{\underline{\theta}}\right)$ $=\sum_{\underline{\theta} \in[\tilde{\underline{\theta}}]}\left|\mathbf{J}\left(\Gamma_{\underline{\theta}}\right)\right|$, and by Lemma $7.3 \operatorname{ker}\left(B(\Gamma) * \overline{\mathscr{S}}_{\hat{\underline{\theta}}}\right)=\mathscr{L}_{\underline{\underline{\hat{\theta}}}}$. Substituting all of these into $(7.10)$ we see that $(7.18)$ is equivalent to $(7 . \overline{17})$.

Finally, when $|[\underline{\tilde{\theta}}]|=0,1$ we know from Corollaries 7.1, 7.3 respectively that $\mathbf{H}_{\underline{\tilde{\theta}}}=\bigoplus_{\underline{\theta} \in[\tilde{\tilde{\theta}}]} \mathscr{H}\left(\Gamma_{\underline{\theta}}\right)$ and therefore such $\underline{\tilde{\theta}}$ cannot satisfy (7.18) or equivalently (7.17). Consequently if $\underline{\tilde{\theta}} \in \tilde{\Theta}(\Gamma)$ satisfies (7.17) we must have $|[\tilde{\theta}]|>1$.

The dimension of $\mathbf{H}(\Gamma)$ can be computed with the aid of the following formulae.

THEOREM 7.3 .

$$
\begin{aligned}
& \operatorname{dim} \mathbf{H}(\Gamma)=\operatorname{dim} \mathscr{S}(\Gamma)-\sum_{\tilde{\tilde{\theta}} \in \tilde{\Theta}(\Gamma)} \operatorname{dim} \mathscr{L}_{\underline{\hat{\theta}}} \\
& =|\mathbf{J}(\Gamma)|-\sum_{\substack{\tilde{\theta} \in \tilde{\tilde{\Theta}}(\Gamma) \\
\mid[\underline{\tilde{\theta}}||>1}}\left(\operatorname{dim} \mathscr{L}_{\underline{\underline{\theta}}}-\left|\mathbf{J}\left(\tilde{\Gamma}_{\underline{\tilde{\theta}}}\right)\right|+\sum_{\underline{\theta} \in[\underline{\tilde{\theta}}]}\left|\mathbf{J}\left(\Gamma_{\underline{\theta}}\right)\right|\right) .
\end{aligned}
$$


PROOF. Since $\mathscr{S}(\Gamma)=\bigoplus_{\underline{\tilde{\theta}} \in \tilde{\Theta}(\Gamma)} \mathscr{S}_{\tilde{\hat{\theta}}}$, the first equation is obtained by summing (7.19) over all $\tilde{\hat{\theta}} \in \tilde{\Theta}(\Gamma)$ and applying Corollary 7.2 and Lemma 7.3. To prove the second equality note that the proof of Theorem 7.2 shows that

$$
\begin{aligned}
\operatorname{dim} \mathscr{H}(\Gamma)-\operatorname{dim} \mathbf{H}(\Gamma) & =\sum_{\underline{\tilde{\theta}} \in \tilde{\Theta}(\Gamma)}\left[\left(\sum_{\underline{\theta} \in[\tilde{\tilde{\theta}}]} \operatorname{dim} \mathscr{H}\left(\Gamma_{\underline{\theta}}\right)\right)-\operatorname{dim} \mathbf{H}_{\underline{\tilde{\theta}}}\right] \\
& =\sum_{\substack{\tilde{\theta} \in \tilde{\Theta}(\Gamma) \\
|[\tilde{\hat{\theta}}]|>1}}\left[\left(\sum_{\underline{\theta} \in[\tilde{\tilde{\theta}}]} \operatorname{dim} \mathscr{H}\left(\Gamma_{\underline{\theta}}\right)\right)-\operatorname{dim} \mathscr{S}_{\underline{\underline{\theta}}}+\operatorname{dim} \mathscr{L}_{\underline{\hat{\theta}}}\right] .
\end{aligned}
$$

Now the desired result follows directly from Theorem 1.1(b).

EXAMPLE 7.1. We use here Theorem 7.2 to show that the sufficient condition for (7.1) established in Theorem 7.1 is not a necessary one.

Let $s=2,|\Gamma|=3,\left(\underline{x}^{1}, \lambda_{1}\right)=((1,0), 0),\left(\underline{x}^{2}, \lambda_{2}\right)=((1,1), 2 \pi i),\left(\underline{x}^{3}, \lambda_{3}\right)=$ $((1,-1), 0)$. In this case $\Gamma$ is simple, $\Theta(\Gamma)=\{(0,0),(0,2 \pi i),(\pi i, \pi i)\}$ and therefore $\mathscr{H}(\Gamma)=\left\langle 1, e^{2 \pi i x_{2}}, e^{\pi i\left(x_{1}+x_{2}\right)}\right\rangle$. On the other hand $\tilde{\Theta}(\Gamma)=\{(0,0),(\pi i, \pi i)\}$ and some straightforward computation shows that

$$
\mathscr{S}_{(0,0)}=\left\langle 1, x_{1}, x_{2}\right\rangle, \quad \mathscr{S}_{(\pi i, \pi i)}=\left\langle e^{\pi i\left(x_{1}+x_{2}\right)}\right\rangle .
$$

Since $|[(\pi i, \pi i)]|=1$, then in view of Theorem 7.2 , in order to verify (7.1) we only have to check $\mathscr{L}_{(0,0)}$. Since $[(0,0)]=\{(0,0),(0,2 \pi i)\}$ then by definition every element of $\mathscr{L}_{(0,0)}$ is a linear polynomial $p$ which satisfies

$$
p(\underline{x})-p(\underline{x}-(1,1))=p(\underline{x})-p(\underline{x}-(1,-1))=0 .
$$

This readily implies that $\mathscr{L}_{(0,0)}=\langle 1\rangle$ and therefore $\operatorname{dim} \mathscr{L}_{(0,0)}=1$. Now $\tilde{\Gamma}_{(0,0)}=\Gamma$ and therefore

$$
\left|\mathbf{J}\left(\tilde{\Gamma}_{(0,0)}\right)\right|=|\mathbf{J}(\Gamma)|=3,
$$

while

$$
\sum_{\underline{\theta} \in[(0,0)]}\left|\mathbf{J}\left(\Gamma_{\underline{\theta}}\right)\right|=\left|\mathbf{J}\left(\Gamma_{(0,0)}\right)\right|+\left|\mathbf{J}\left(\Gamma_{(0,2 \pi i)}\right)\right|=1+1=2 .
$$

Thus we see that (7.17) is not valid here and Theorem 7.2 implies $\mathbf{H}(\Gamma)=\mathscr{H}(\Gamma)$. Indeed, it can be shown (e.g., by Poisson's summation formula) that

$$
\begin{gathered}
B(\Gamma) *^{\prime}\left(x_{1}+x_{2}\right)=2, \quad B(\Gamma) *^{\prime}\left(x_{1}-x_{2}\right)=2 e^{2 \pi i x_{2}}, \\
B(\Gamma) *^{\prime} e^{\pi i\left(x_{1}+x_{2}\right)}=-4 e^{\pi i\left(x_{1}+x_{2}\right)} .
\end{gathered}
$$

To make use of Theorem 7.2 one needs to compute $\operatorname{dim} \mathscr{L}_{\underline{\underline{\theta}}}$ at least for cases when $|[\tilde{\theta}]|>1$. In the following we show that Theorem 7.2 implies a necessary condition for (7.1) based only on the structure of $\Theta(\Gamma)$.

THEOREM 7.4. Assume that there exists $\underline{\tilde{\theta}} \in \tilde{\Theta}(\Gamma)$ and $K \subset \Gamma$ such that

(i) $|\Theta(K)|>1$,

(ii) $\Theta(K) \subset[\tilde{\theta}]$.

Then $\mathbf{H}(\Gamma)$ is a proper subspace of $\mathscr{H}(\Gamma)$. 
Proof. First assume $\Gamma=K$. Since $\Theta(\Gamma) \subset[\underline{\tilde{\theta}}]$ it follows that $\tilde{\Gamma}_{\tilde{\underline{\theta}}}=\Gamma$. But since $|\Theta(\Gamma)|>1$ then for each $\underline{\theta} \in \Theta(\Gamma) \Gamma \backslash \Gamma_{\underline{\theta}} \neq \varnothing$ and therefore $\nabla^{\tilde{\tilde{\Gamma}_{\underline{\theta}}}} \bar{\Gamma}_{\underline{\underline{\theta}}}=\nabla^{\Gamma \backslash \Gamma_{\underline{\theta}}}$ annihilates $e_{\underline{\tilde{\theta}}} \in \mathscr{S}_{\underline{\underline{\hat{\theta}}}}$. This means that $\operatorname{dim} \mathscr{L}_{\underline{\tilde{\theta}}} \geq 1$. On the other hand

$$
\left|\mathbf{J}\left(\tilde{\Gamma}_{\tilde{\tilde{\theta}}}\right)\right|-\sum_{\underline{\theta} \in[\tilde{\tilde{\theta}}]}\left|\mathbf{J}\left(\Gamma_{\underline{\theta}}\right)\right|=|\mathbf{J}(\Gamma)|-\sum_{\underline{\theta} \in \Theta(\Gamma)}\left|\mathbf{J}\left(\Gamma_{\underline{\theta}}\right)\right|=0 .
$$

Consequently $\underline{\tilde{\theta}}$ satisfies (7.17) and the claim follows in this case from Theorem 7.2. If $K \varsubsetneqq \Gamma$ (and $\langle K\rangle=\mathbb{R}^{s}$ ) then by the proof above $\mathbf{H}(K) \neq \mathscr{H}(K)$. Choose $f \in \mathscr{H}(K) \backslash \mathbf{H}(K)$. By Lemma 7.2 there exists $g \in \mathscr{H}(\Gamma)$ such that $D^{\Gamma \backslash K} g=f$. We claim that $g \notin \mathbf{H}(\Gamma)$. Indeed, if $g \in \mathbf{H}(\Gamma)$ then $g=B(\Gamma) * u$ where $u(\cdot)$ is some sequence defined on $\mathbb{Z}^{s}$. In this case (1.15) would imply

$$
f=D^{\Gamma \backslash K} g=D^{\Gamma \backslash K}(B(\Gamma) * s)=B(K) * \nabla^{\Gamma \backslash K} u,
$$

contradicting the fact that $f \notin \mathbf{H}(K)$. Consequently $g \in \mathscr{H}(\Gamma) \backslash \mathbf{H}(\Gamma)$ and the proof of Theorem 7.4 is completed.

Finally, we note that in the univariate case condition (7.13) is necessary as well:

COROLLARY 7.4. In case $s=1,(7.13)$ is a necessary and sufficient condition for (7.1).

PROOF. The sufficiency has been proved in Theorem 7.1. To verify the necessity note that in the present situation each $\gamma \in \Gamma$ is a basis in $\mathbf{J}(\Gamma)$ and therefore determines a unique $\theta_{\gamma} \in \Theta(\Gamma)$. Now, if (7.13) is not valid then there exists $\tilde{\theta} \in \tilde{\Theta}(\Gamma)$ and distinct $\theta^{1}, \theta^{2} \in \Theta(\Gamma)$ such that $\left\{\theta^{1}, \theta^{2}\right\} \subset[\tilde{\theta}]$. Choose $\left\{\gamma_{1}, \gamma_{2}\right\} \subset \Gamma$ such that $\theta_{\gamma_{j}}=\theta^{j}, j=1,2$. We conclude that $\Theta\left(\left\{\gamma_{1}, \gamma_{2}\right\}\right)=\left\{\theta^{1}, \theta^{2}\right\} \subset[\tilde{\theta}]$. Application of Theorem 7.4 with $K=\left\{\gamma_{1}, \gamma_{2}\right\}$ shows therefore that (7.1) does not hold.

\section{REFERENCES}

[BD] C. de Boor and R. DeVore, Approximation by smooth multivariate splines, Trans. Amer. Math. Soc. 276 (1983), 775-788.

[BH] C. de Boor and K. Höllig, B-splines from parallelepipeds, J. Analyse Math. 42 (1982/1983), 99-115.

[DM $\mathbf{D}_{1}$ W. Dahmen and C. A. Micchelli, Translates of multivariate splines, Linear Algebra and Appl. 52/53 (1983), 217-234.

$\left[\mathbf{D M}_{2}\right] \ldots$, On the local linear independence of translates of a box spline, Studia Math. 82 (1985), 243-263.

$\left[\mathbf{D M}_{3}\right] \ldots$, On the solution of certain systems of partial difference equations and linear dependence of translates of box splines, Trans. Amer. Math. Soc. 292 (1985), 305-320.

[DR] N. Dyn and A. Ron, Local approximation by certain spaces of exponential polynomials, approximation order for exponential box splines and related interpolation problems, CAT Rep. 160, Texas A\&M Univ., College Station, Tex. (January 1988).

[G] D. I. Gurevich, Counterexamples to the problem of L. Schwartz, Functional Anal. Appl. 9 (1975), 116-120.

[ $\left.\mathbf{J}_{1}\right]$ R. Q. Jia, On the linear independence of translates of box splines, J. Approx. Theory 40 (1984), 158-160.

$\left[\mathbf{J}_{2}\right] \ldots$, , Local linear independence of the translates of a box spline, Constr. Approx. 1 (1985), 175-182.

[L] S. Lang, Algebra, Addison-Wesley 1965.

$\left[\mathbf{R}_{1}\right]$ A. Ron, Exponential box splines, Constr. Approx. (to appear). 
$\left[\mathbf{R}_{2}\right] \ldots$, Linear independence for the translates of an exponential box, Rocky Mountain J. Math. (to appear).

[Ru] W. Rudin, Functional analysis, McGraw-Hill, 1973.

[S $\left.\mathbf{S}_{1}\right]$ L. Schwartz, Théorie générale des fonctions moyenne-periodiques, Ann. of Math. (2) 48 (1947), $857-929$.

$\left[\mathbf{S}_{2}\right] \ldots$, Analyse et synthése harmoniques dans les espaces de distributions, Canad. J. Math. 3 (1951), 503-512.

SCHOOL OF Mathematical SCIENCEs, Raymond and BeVerly SaCKLER FaCUlty OF EXACT SCIENCES, TEL-AVIV UNIVERSITY, TEL-AVIV, ISRAEL

Current address (Amos Ron): Computer Sciences Department, University of Wisconsin-Madison, 1210 West Dayton Street, Madison, Wisconsin 53706

Current address (Asher Ben-Artzi): Laboratory for Mathematics and Statistics, University of California at San Diego, La Jolla, California 92093-0003 